\title{
Some new thin sets of integers in Harmonic Analysis
}

\author{
Daniel Li, \\ Hervé Queffélec, Luis Rodríguez-Piazza
}

\begin{abstract}
We randomly construct various subsets $\Lambda$ of the integers which have both smallness and largeness properties. They are small since they are very close, in various meanings, to Sidon sets: the continuous functions with spectrum in $\Lambda$ have uniformly convergent series, and their Fourier coefficients are in $\ell_{p}$ for all $p>1$; moreover, all the Lebesgue spaces $L_{\Lambda}^{q}$ are equal for $q<+\infty$. On the other hand, they are large in the sense that they are dense in the Bohr group and that the space of the bounded functions with spectrum in $\Lambda$ is non separable. So these sets are very different from the thin sets of integers previously known.

Résumé. On construit aléatoirement des ensembles $\Lambda$ d'entiers positifs jouissant simultanément de propriétés qui les font apparâ̂tre à la fois comme petits et comme grands. Ils sont petits car très proches à plus d'un égard des ensembles de Sidon: les fonctions continues à spectre dans $\Lambda$ ont une série de Fourier uniformément convergente, et ont des coefficients de Fourier dans $\ell_{p}$ pour tout $p>1$; de plus, tous les espaces de Lebesgue $L_{\Lambda}^{q}$ coïncident pour $q<+\infty$. Mais ils sont par ailleurs grands au sens où ils sont denses dans le compactifié de Bohr et où l'espace des fonctions bornées à spectre dans $\Lambda$ n'est pas séparable. Ces ensembles sont donc très différents des ensembles minces d'entiers connus auparavant.
\end{abstract}

Key-words. ergodic set - lacunary set $-\Lambda(q)$-set - quasi-independent set - random set $-p$-Rider set - Rosenthal set $-p$-Sidon set - set of uniform convergence - uniformly distributed set.

Mathematics Subject Classification. Primary: 42A36 - 42A44 - 42A55 42A61 - 43A46; Secondary: 60D05

\section{Introduction}

It is well known that the Fourier series of an integrable function defined on the unit-circle $\mathbb{T}=\mathbb{R} / 2 \pi \mathbb{Z}$ of the complex plane $\mathbb{C}$ can be badly behaved. For example, it is well known that there exist continuous functions whose Fourier series is not everywhere convergent (see [30], Th. 18.1, and Th. 19.5 for the optimal result), and integrable ones with everywhere divergent Fourier series (see [30], Th. 19.2 for instance; see also [29]).

The problem of thin sets of integers is the following: instead of considering all the integrable functions on $\mathbb{T}$, or all the continuous ones, we consider only 
those whose spectrum (the set where their Fourier coefficients do not vanish) is contained in a prescribed subset $\Lambda$ of the integers $\mathbb{Z}$. This set $\Lambda$ will be said "thin" if the Fourier series of these functions behaves better than in the general case. A typical example is $\Lambda=\left\{1,3,3^{2}, \ldots, 3^{n}, \ldots\right\}$. It is well known (see 62], for instance) that every integrable function $f$ with spectrum in $\Lambda\left(f \in L_{\Lambda}^{1}\right)$ is actually square integrable, and that every continuous function $f$ with spectrum in $\Lambda\left(f \in \mathcal{C}_{\Lambda}\right)$ has a normally convergent Fourier series (equivalently $\left.\widehat{f} \in \ell_{1}\right)$.

In his seminal paper [54, W. Rudin defined two notions of thinness for $\Lambda: \Lambda$ is a Sidon set if $f \in \mathcal{C}_{\Lambda}$ implies that $\widehat{f} \in \ell_{1}$, and $\Lambda$ is a $\Lambda(q)$-set for some $q>1$, if $f \in L_{\Lambda}^{1}$ implies that $f \in L^{q}$. These concepts may as well be defined in the more general setting of a compact abelian group $G$ equipped with its normalized Haar measure, and for a subset $\Lambda$ of its discrete dual group $\Gamma$.

$\mathrm{W}$. Rudin studied the general properties of those sets and the connection between the two notions. In particular, he showed that Sidon sets are $\Lambda(q)$-sets for all $q<+\infty$, and that, more precisely:

(0.1) $\Lambda$ Sidon implies $\|f\|_{q} \leq C \sqrt{q}\|f\|_{2}$ for every $\Lambda$-polynomial $f$ and for every $q \geq 2$, where $C$ is a constant which depends only on the Sidon constant of $\Lambda$.

Since then, several new notions of thin sets emerged. These include $p$-Sidon sets (see [2], 3], 44, 5], [14], [18, [21], 23], 24], [34, 447, 60]), and sets of uniform convergence (see [1], [19], [20], [21], [26], [33], [44], [45], [56], [57]): every continuous function with spectrum in such a set has its Fourier seriesin $\ell_{p}$ or uniformly convergent, respectively. But the examples of such sets were always nearly the same: products (sometimes "fractional products": [3], [4, [5]), or sums of Sidon sets, which is a severe restriction for the geometry of the Banach space $\mathcal{C}_{\Lambda}$. For example, F. Lust-Piquard (40]) proved that:

(0.2) The injective tensor product $\ell_{1} \widehat{\otimes}_{\varepsilon} \cdots \widehat{\otimes}_{\varepsilon} \ell_{1}$ has the Schur property (i.e. weakly null sequences converge in norm to zero).

It follows easily that:

(0.3) If $\Lambda=E_{1} \times \cdots \times E_{k}$, where the $E_{j}$ 's are Sidon sets, then $\mathcal{C}_{\Lambda}$ has the Schur property; in particular, $\mathcal{C}_{\Lambda}$ does not contain $c_{0}$, the space of sequences going to zero at infinity.

Since these sets were essentially the only known examples of $p$-Sidon sets (they are exactly $2 N /(N+1)$-Sidon), one could believe that all $p$-Sidon sets have this property. It should be mentionned that in [3], R. Blei constructed for each $p \in] 1,2$ [, exactly $p$-Sidon sets, using fractional products, so of a different type, but the corresponding space $\mathcal{C}_{\Lambda}$ appears as an $\ell_{1}$-sum of finite dimensional spaces, and so does have the Schur property (we thank R. Blei for this remark).

Because of this lack of examples, the comparison between two classes of thin sets proved to be very difficult: whether a $p$-Sidon, or a set of uniform convergence is a $\Lambda(q)$-set for some $q>1$ is still an open problem. On the other 
hand, considerable progress concerning the Sidon sets or $\Lambda(q)$-sets has been made: for example, G. Pisier ([47], Th. 6.2) proved that the converse of (0.1) is true, and J. Bourgain ([12]) proved that for each $q>2$ there exist "exactly" $\Lambda(q)$-sets, i.e. sets which are $\Lambda(q)$, but $\Lambda\left(q^{\prime}\right)$ for no $q^{\prime}>q$. Both authors used random methods, and more specifically, J. Bourgain popularized the "method of selectors" to produce several thin sets $\Lambda$ with unusual properties, such as being "uniformly distributed", which implies, by a result of F. Lust-Piquard ([42]), that $\mathcal{C}_{\Lambda}$ contains $c_{0}$ and therefore is not a Rosenthal set (i.e. there are bounded measurable functions with spectrum in $\Lambda$ wich are not almost everywhere equal to a continuous function), and which also implies that $\Lambda$ is dense in the Bohr group (see [6], Theorem 1). This allowed the first named author to see that there are sets of integers which are $\Lambda(q)$ for all $q<+\infty$ but not Rosenthal ([37]; see also [43]).

The aim of this paper is the construction of random sets $\Lambda$ of integers which have thinness properties, but wich are not Rosenthal sets (i.e. $\mathcal{C}_{\Lambda}$ is not the whole $L_{\Lambda}^{\infty}$ ), actually such that $\mathcal{C}_{\Lambda}$ contains $c_{0}$, and are dense in the Bohr group. In view of (0.3), these sets will necessarily be very exotic compared to the previously known examples. This shows that replacing absolute convergence of the Fourier series by uniform convergence (sets of uniform convergence) or by $\ell_{p}$ convergence for $p>1$ ( $p$-Sidon sets) gives sets which are very far from Sidon sets. This constrasts with Pisier's result saying that $\Lambda$ is necessarily a Sidon set whenever $\widehat{f} \in \ell_{1, \infty}$ for every $f \in \mathcal{C}_{\Lambda}$ (from [48], Théorème 2.3 (vi), and the top of page 688). On the other hand, though non-Sidon Rosenthal sets do exist ([53]), it follows from Bourgain-Milman's cotype theorem ([13]) that, for every non-Sidon set $\Lambda, \mathcal{C}_{\Lambda}$ does contain $\ell_{\infty}^{n}$ uniformly, so that the presence of $c_{0}$ inside $\mathcal{C}_{\Lambda}$ for non-Sidon $\Lambda$ may appear not so surprising. Although it is not known whether Sidon sets may be dense in the Bohr group, we obtain in this paper, as mentioned above, sets which are dense in the Bohr group, and are of uniform convergence and $p$-Sidon for every $p>1$.

We construct essentially four types of sets. Each of them will be a non Rosenthal set, but a set of uniform convergence, $\Lambda(q)$ for all $q<+\infty$, and with moreover additional properties of $p$-Sidonicity.

The first one (Theorem 2.2) is a very lacunary set $\Lambda$ with the nicest properties: it is $p$-Sidon for all $p>1$. The second and third ones (Theorem 2.5 and Theorem 2.6) are medium lacunary sets: for each $p$ with $1<p<4 / 3$, they are, in Theorem 2.5, $p$-Rider (a weaker property than being $p$-Sidon, see the definition below), but not $q$-Rider for $q<p$, and are $q$-Sidon for every $q>p /(2-p)$; and in Theorem 2.6, they are $q$-Rider for every $q>p$, but not $p$-Rider, and they are $q$-Sidon for every $q>p /(2-p)$. Finally, the fourth type (Theorem 2.7) is a set $\Lambda$ which is, in some sense as little lacunary as possible if we want its trace on each interval $[N, 2 N[$ to have a bounded Sidon constant. It leads to sets which are 4/3-Rider, but not $q$-Rider for $q<4 / 3$.

We construct these sets by using various choices of selectors, and adding arithmetical, functional or probabilistic arguments. The treatment of the last case requires a different probabilistic approach, taken from [8]. 
It should be noted that in the two first cases the sets are uniformly distributed; in the fourth case, however, the sets $\Lambda$ only have positive upper density in uniformly distributed sets. Nevertheless, $\mathcal{C}_{\Lambda}$ still contains $c_{0}$, by a result of F. Lust-Piquard (42], Th. 5).

Acknowledgement. Part of this paper was made when the first named author was a guest of the Departamento de Análisis Matemático de la Universidad de Sevilla in April 1999, and when the third named author was a guest of the Université d'Artois in Lens in june 1999.

\section{Notation, definitions and preliminary results}

We denote by $\mathbb{T}$ the compact abelian group of complex numbers of modulus one, equipped with its normalized Haar measure $m . \mathcal{C}(\mathbb{T})$ denotes the space of continuous complex functions defined on $\mathbb{T}$, equipped with its sup norm \|\|$_{\infty}$ and identified as usual with the space of continuous $2 \pi$-periodic complex functions defined on $\mathbb{R}$. If $\Lambda$ is a subset of the dual group $\mathbb{Z}, \mathcal{C}_{\Lambda}$ will denote the subspace of $\mathcal{C}(\mathbb{T})$ consisting of functions whose spectrum lies in $\Lambda$ :

$$
\widehat{f}(n) \equiv \int_{\mathbb{T}} f e_{-n} d m=0 \quad \text { if } n \in \mathbb{Z} \backslash \Lambda,
$$

where $e_{n}(z)=z^{n}$, or equivalently, $e_{n}(t)=\mathrm{e}^{i n t}$.

$\mathcal{C}_{\Lambda}$ is the uniform closure of the space $\mathcal{P}_{\Lambda}$ of trigonometric polynomials with spectrum in $\Lambda$, i.e. the uniform closure of the subspace $\mathcal{P}_{\Lambda}$ generated by the characters $e_{n}$, with $n \in \Lambda$.

For $f \in \mathcal{C}(\mathbb{T}), 1 \leq q<+\infty, M$ and $N$ positive integers, we shall denote the Fourier sums of $f$ by:

$$
S_{M, N}(f)=\sum_{-M}^{N} \widehat{f}(n) e_{n}
$$

and the symmetric Fourier sums of $f$ by:

$$
S_{N}(f)=S_{N, N}(f)=\sum_{-N}^{N} \widehat{f}(n) e_{n} .
$$

$|A|$ denotes the cardinality of the finite set $A$.

A relation in $\Lambda \subseteq \mathbb{Z}^{*} \equiv \mathbb{Z} \backslash\{0\}$ is a $(+1,-1,0)$-valued sequence $\left(\theta_{k}\right)_{k \in \Lambda}$ such that $\sum\left|\theta_{k}\right|<+\infty$ and $\sum \theta_{k} k=0$. The set $S=\left\{k ; \theta_{k} \neq 0\right\}$ is called the support of the the relation, and $|S|=\sum\left|\theta_{k}\right|$ is called its length.

The relation $\left(\theta_{k}^{\prime}\right)_{k \in \Lambda}$ is said to be longer than the relation $\left(\theta_{k}\right)_{k \in \Lambda}$ if $\theta_{k} \neq 0$ implies $\theta_{k}=\theta_{k}^{\prime}$.

The set $\Lambda \subseteq \mathbb{Z}^{*}$ is quasi-independent if it contains no non-trivial relation (i.e. with non-empty support). Typically, $\Lambda=\left\{1,2,4, \ldots, 2^{n}, \ldots\right\}$ is quasiindependent. The quasi-independent sets are the prototype of Sidon sets, i.e. 
of sets $\Lambda$ for which: $\|\widehat{f}\|_{1} \leq K\|f\|_{\infty}$ for all $f \in \mathcal{C}_{\Lambda}$. The best constant $K$ in this inequality is called the Sidon constant of $\Lambda$ and is denoted by $S(\Lambda)$. We will refer to [39] for standard notions on Sidon sets. It is known that quasi-independent sets are not only Sidon sets but their Sidon constant is bounded by an absolute constant: this follows from [54], Th. 2.4 and [49, Lemma 1.7. Other proofs can be found in [48], lemme 3.2, and in [9], Prop. 1. We shall use the fact that $S(\Lambda) \leq 8$ if $\Lambda$ is quasi-independent.

Let us recall now some classical definitions and results.

A set $\Lambda \subseteq \mathbb{Z}$ is said to be a $\Lambda(q)$-set (where $q>2$ ) if there exists a positive constant $C_{q}$ such that $\|f\|_{q} \leq C_{q}\|f\|_{2}$ for every $f \in \mathcal{P}_{\Lambda}$.

The notion of a $\Lambda(q)$-set is, in some sense, local. That follows from the Littlewood-Paley theory. The next proposition is essentially well-known, except for the growth of the constant, for which we have found no reference. Accordingly, we offer a short proof.

Proposition 1.1 Let $\Lambda \subseteq[2,+\infty[$. Then:

(a) Let $\left(M_{n}\right)_{n \geq 1}$ be a sequence of positive integers such that $M_{1} \leq 2$ and $M_{n+1} / M_{n} \geq \alpha>1$. If $\Lambda \cap\left[M_{n}, M_{n+1}[, n \geq 1\right.$, has a uniformly bounded Sidon constant, then $\Lambda$ is $\Lambda(q)$ for all $q \geq 2$; more precisely: $\|f\|_{q} \leq C(q, \alpha)\|f\|_{2}$ for every $f \in \mathcal{P}_{\Lambda}$.

(b) If $\Lambda \cap\left[2^{n}, 2^{n+1}[, n \geq 1\right.$, has a uniformly bounded Sidon constant, $\Lambda$ is $\Lambda(q)$ for every $q \geq 2$ and, more precisely: $\|f\|_{q} \leq C q^{2}\|f\|_{2}$ for every $f \in \mathcal{P}_{\Lambda}$ and for some numerical constant $C$.

Proof. (a) Set

$$
f_{k}=\sum_{M_{k} \leq n<M_{k+1}} \widehat{f}(n) e_{n} \quad \text { and } \quad S f=\left(\sum_{k=1}^{+\infty}\left|f_{k}\right|^{2}\right)^{1 / 2} .
$$

Since $M_{k+1} / M_{k} \geq \alpha>1$ and $\Lambda \subseteq\left[M_{1},+\infty\right.$ [, we have (62], Chap. XV, Th. 2.1):

$$
\|f\|_{q} \leq C_{0}(q, \alpha)\|S f\|_{q} .
$$

Now, using the 2-convexity of the $L^{q}$-norm for $q \geq 2$, we obtain:

$$
\|S f\|_{q} \leq\left(\sum_{k=1}^{+\infty}\left\|f_{k}\right\|_{q}^{2}\right)^{1 / 2} .
$$

But $f_{k} \in \mathcal{P}_{\Lambda_{k}}$, where $\Lambda_{k}=\Lambda \cap\left[M_{k}, M_{k+1}\right.$ [ has a uniformly bounded Sidon constant. Therefore $\left\|f_{k}\right\|_{q} \leq C_{1} \sqrt{q}\|f\|_{2}$, where $C_{1}$ is a numerical constant. The result follows.

(b) We now make use of the classical square function

$$
S g=\left(\sum_{k \in \mathbb{Z}}\left|g_{k}\right|^{2}\right)^{1 / 2},
$$


where

$g_{k}=\sum_{2^{k} \leq n<2^{k+1}} \widehat{g}(n) e_{n}$, if $k \geq 0 \quad$ and $\quad g_{k}=\sum_{-2^{|k|+1}<n \leq-2^{|k|}} \widehat{g}(n) e_{n}$ if $k<0$.

For this classical square function, we have the following sharp inequality, due to J. Bourgain ([11, Th. 1):

$$
\|S g\|_{p} \leq C_{0}(p-1)^{-3 / 2}\|g\|_{p} \quad \text { for } 1<p \leq 2,
$$

where $C_{0}$ is a numerical constant. We deduce by duality that:

$$
\|f\|_{q} \leq C_{0} q^{3 / 2}\|S f\|_{q} \quad \text { for } 2 \leq q<+\infty .
$$

In fact, by orthogonality (recall that $f \in \mathcal{P}_{\Lambda}$ and that $\Lambda \subseteq[2,+\infty[$ ) and the Cauchy-Schwarz inequality, we have, for every $g \in L^{p}$ with $\|g\|_{p}=1(1 / p+$ $1 / q=1)$ :

$$
\begin{aligned}
|<f, g>|=\mid & \sum_{k=1}^{+\infty}<f_{k}, g_{k}>|=| \int_{\mathbb{T}} \sum_{k=1}^{+\infty} f_{k}(-t) g_{k}(t) d m(t) \mid \\
& \leq \int_{\mathbb{T}} S f(-t) S g(t) d m(t) \\
& \leq\|S f\|_{q}\|S g\|_{p} \leq C_{0}(p-1)^{-3 / 2}\|S f\|_{q} \\
& \leq C_{0} q^{3 / 2}\|S f\|_{q} .
\end{aligned}
$$

This means that here we are allowed to take $C_{0}(q, 2)=C_{0} q^{3 / 2}$ in part (a) of the proof. The rest is unchanged, and we can also take $C(q, 2)=C_{1} \sqrt{q} C_{0} q^{3 / 2}=$ $C q^{2}$.

A set $\Lambda \subseteq \mathbb{Z}$ is called a set of uniform convergence (in short a $U C$-set) if, for any $f \in \mathcal{C}_{\Lambda}$, the symmetric Fourier sums $S_{N}(f)$ converge uniformly to $f$. Its constant of uniform convergence $U(\Lambda)$ is the smallest constant $K$ such that, for any $f \in \mathcal{C}_{\Lambda}$ :

$$
\sup _{N}\left\|S_{N}(f)\right\|_{\infty} \leq K\|f\|_{\infty} .
$$

The following variant turns out to be more tractable ([56]). $\Lambda$ is called a set of complete uniform convergence (in short a CUC-set) if the translates $(\Lambda+a)$ are uniformly UC for $a \in \mathbb{Z}$, or equivalently, if the Fourier sums $S_{M, N}(f)$ converge uniformly to $f$ as $M, N$ go to $+\infty$, for every $f \in \mathcal{C}_{\Lambda}$.

The two notions turn out to be distinct ([20]), but clearly coincide if $\Lambda \subseteq \mathbb{N}$, which will always be the case in the sequel. The notion of CUC-set is also a local one as the following proposition shows.

Proposition 1.2 ([57], Th. 3) Let $\Lambda \subseteq \mathbb{N}^{*}$ and $\Lambda_{N}=\Lambda \cap[N, 2 N[$.

(a) If $U\left(\Lambda_{N}\right)$ is bounded by $K$ for $N=1,2, \ldots$, then $\Lambda$ is a CUC-set.

(b) Let $\left(M_{n}\right)_{n \geq 1}$ be a sequence of positive integers such that $M_{n+1} / M_{n} \geq 2$. Then, if $\Lambda \cap\left[M_{n}, M_{n+1}\right.$ [ are quasi-independent for each $n$, or more generally if they are Sidon sets with uniformly bounded Sidon constant, then $\Lambda$ is a CUC-set. 
Remark. (b) is a useful criterion to produce sets that are CUC but not Sidon; for instance, if $\Lambda=\bigcup_{n=1}^{+\infty}\left\{2^{n}+2^{j} ; j=0, \ldots, n-1\right\}$, then $\Lambda \cap\left[2^{n}, 2^{n+1}\right.$ [ is quasi-independent, whereas $\Lambda \cap[1, N]$ has about $(\log N)^{2}$ elements, and therefore cannot be Sidon (the mesh condition for Sidon sets, see Proposition 1.6 below, is violated).

The random variables which we shall use will always be defined on some probability space $(\Omega, \mathcal{A}, \mathbb{P})$ which will play no explicit role, and the expectation with respect to $\mathbb{P}$ will always be denoted by $\mathbb{E}$ :

$$
\mathbb{E}(X)=\int_{\Omega} X(\omega) d \mathbb{P}(\omega) .
$$

Recall the (more or less) classical deviation inequality (see [32], § 6.3):

Lemma 1.3 Let $X_{1}, \ldots, X_{N}$ be independent centered complex random variables such that $\left|X_{k}\right| \leq 1, k=1, \ldots, N$. Let $\sigma \geq \sum_{k=1}^{N} \mathbb{E}\left|X_{k}\right|^{2}$. Then, one has, for every $a \leq \sigma:$

$$
\mathbb{P}\left(\left|X_{1}+\cdots+X_{N}\right| \geq a\right) \leq 4 \exp \left(-a^{2} / 8 \sigma\right) .
$$

Let $\left(r_{n}\right)_{n}$ be a Bernoulli sequence, i.e. a sequence of independent random variables such that:

$$
\mathbb{P}\left(r_{n}=1\right)=\mathbb{P}\left(r_{n}=-1\right)=1 / 2 .
$$

For $f \in \mathcal{P}$, the space of trigonometric polynomials, $\llbracket f \rrbracket$ denotes the norm of $f$ in the Pisier's space $\mathcal{C}^{a . s .}$ :

$$
\llbracket f \rrbracket=\mathbb{E}\left\|\sum_{n} r_{n} \widehat{f}(n) e_{n}\right\|_{\infty} .
$$

See [25] and [47] for more information about this norm.

Definition $1.4 A$ set $\Lambda \subseteq \mathbb{Z}$ is called a $p$-Sidon set $(1 \leq p<2)$ if there exists a constant $K$ such that $\|\widehat{f}\|_{p} \leq K\|f\|_{\infty}$ for all $f \in \mathcal{P}_{\Lambda}$.

It is said to be a p-Rider set if there exists a constant $K$ such that $\|\widehat{f}\|_{p} \leq$ $K \llbracket f \rrbracket$ for all $f \in \mathcal{P}_{\Lambda}$.

$p$-Rider sets were implicitely introduced, with different definition, in 18 (Th. 2.4), and in [23], p. 213, as class $\mathcal{T}_{p}$ (see also [47], Th. 6.3). They were explicitely defined and studied in [51] and [52] under the name " $p$-Sidon presque sûrs". We used "almost surely $p$-Sidon set" in the first version of this paper, but, following a suggestion of J.-P. Kahane, we now use the terminology " $p$-Rider".

Clearly, every $p$-Sidon set is $p$-Rider. The converse is true for $p=1$ : this is a remarkable result due to D. Rider ([50]), making clever use of Drury's convolution device (which proves that the union of two Sidon sets is Sidon [17]). Whether this converse is still true for $1<p<2$ is an open problem. 
Definition 1.5 We shall say that a finite set $B \subseteq \Lambda$ is $M$-pseudo-complemented in $\Lambda$ if there exists a measure $\mu$ on $\mathbb{T}$ such that:

$$
|\widehat{\mu}| \geq 1 \text { on } B ; \quad \widehat{\mu}=0 \text { on } \Lambda \backslash B ; \quad\|\mu\| \leq M .
$$

The following proposition gives some necessary, sufficient, or necessary and sufficient conditions for a set $\Lambda$ to be $p$-Sidon or $p$-Rider. Part (b) of this proposition seems to be new.

Proposition 1.6 Let $\Lambda \subseteq \mathbb{Z}^{*}$ and $1 \leq p<2$. Set $\varepsilon(p)=2 / p-1$. Then:

(a) $\Lambda$ is a p-Rider set if and only if there exists a constant $\delta>0$ such that, for every finite set $A \subseteq \Lambda$, there exists a quasi-independent subset $B \subseteq A$ such that $|B| \geq \delta|A|^{\varepsilon(p)}$.

(b) Let $q_{0}>1$. If there exists a constant $\delta>0$ such that, for every finite set $A \subseteq \Lambda$, there exists a quasi-independent subset $B \subseteq A$ such that $|B| \geq \delta|A|^{1 / q_{0}}$ and if $B$ can moreover be taken $M$-pseudo-complemented in $\Lambda$, for some fixed $M$, then $\Lambda$ is a $q$-Sidon set for every $q>q_{0}$.

(c) If $\Lambda$ is a p-Rider set, we have the following mesh condition:

$$
|\Lambda \cap[1, N]| \leq C(\log N)^{p /(2-p)} .
$$

Proof. We refer to [51] for the proof of (a) and (c). To prove (b), let $f \in \mathcal{P}_{\Lambda}$, fix $t>0$, and set $A=\{|\widehat{f}|>t\}$. Take $B \subseteq A$ and $\mu$ as in Definition 1.4. Then $B$ is a Sidon set with Sidon constant $\leq 8$, and since $f * \mu=\sum_{n \in B} \widehat{f}(n) \widehat{\mu}(n) e_{n}$,

$$
\begin{aligned}
\|f\|_{\infty} \geq M^{-1}\left\|\sum_{B} \widehat{f}(n) \widehat{\mu}(n) e_{n}\right\|_{\infty} & \geq \frac{1}{8 M} \sum_{B}|\widehat{f}(n)||\widehat{\mu}(n)| \\
& \geq \frac{1}{8 M} \sum_{B}|\widehat{f}(n)| \geq \frac{t|B|}{8 M} \geq \frac{t \delta|A|^{1 / q_{0}}}{8 M} .
\end{aligned}
$$

In other words, for some constant $C>0$, one has:

$$
t .|\{|\widehat{f}|>t\}|^{1 / q_{0}} \leq C\|f\|_{\infty}, \quad \text { for every } t>0,
$$

which means that the Lorentz norm of $\widehat{f}$ in the Lorentz space $\ell_{q_{0}, \infty}$ is dominated by $\|f\|_{\infty}$.

Now, $\ell_{q_{0}, \infty}$ is continuously injected in $\ell_{q}$ for $q>q_{0}$ (see for instance [38, II p. 143), and this gives the desired result.

We denote, as usual, by $c_{0}$ the classical space of sequences $x=\left(x_{n}\right)_{n \geq 0}$ tending to zero at infinity, equipped with the norm $\|x\|=\sup _{n}\left|x_{n}\right|$. We say, in the usual familiar way, that a Banach space $X$ "contains $c_{0}$ " if $X$ has a closed subspace isomorphic to $c_{0}$. Our notation for Banach spaces is classical, as can be found in [16], [38] or [59] for instance.

A subset $\Lambda$ of $\mathbb{Z}$ is said to be a Rosenthal set if every bounded measurable function on $\mathbb{T}$ with spectrum in $\Lambda$ is almost everywhere equal to a continuous 
function (in short $L_{\Lambda}^{\infty}=\mathcal{C}_{\Lambda}$ ). $\Lambda$ is not Rosenthal if and only if $L_{\Lambda}^{\infty}$ is not separable, so such a set can be thought as being a big set.

Every Sidon set is clearly Rosenthal, but H.P. Rosenthal gave examples of non-Sidon sets which are Rosenthal ([53]). We shall make use of the following well known negative criterion (see [41], §3), which follows from the classical theorem of C. Bessaga and A. Pełczyński ([38], I.2.e.8), saying that a dual space which contains $c_{0}$ has to contain also $\ell_{\infty}$.

Proposition 1.7 If $\mathcal{C}_{\Lambda}$ contains $c_{0}$, then $\Lambda$ is not a Rosenthal set.

Definition 1.8 Let $\Lambda \subseteq \mathbb{N}^{*} \equiv \mathbb{N} \backslash\{0\}$, and set

$$
\Lambda_{N}=\Lambda \cap[1, N] \quad \text { and } \quad A_{N}(t)=\frac{1}{\left|\Lambda_{N}\right|} \sum_{n \in \Lambda_{N}} e_{n}(t) .
$$

We say that $\Lambda$ is:

- ergodic if $\left(A_{N}(t)\right)_{N \geq 1}$ converges to a limit $l_{\Lambda}(t) \in \mathbb{C}$ for each $t \in \mathbb{T}$.

- strongly ergodic if it is ergodic and moreover the limit function $l_{\Lambda}$ defines an element of $c_{0}(\mathbb{T})$ : for every $\varepsilon>0$ the set $\left\{t \in \mathbb{T} ;\left|l_{\Lambda}(t)\right|>\varepsilon\right\}$ is finite.

- uniformly distributed if it is (strongly) ergodic and, moreover, $l_{\Lambda}(t)=0$ for $t \neq 0$ mod. $2 \pi$.

The reason for this terminology is that the ergodic sets are those for which an ergodic theorem holds: $\left(1 /\left|\Lambda_{N}\right|\right) \sum_{n \in \Lambda_{N}} T^{n}$ converges in the strong operator topology for every contraction $T$ of a Hilbert space. Typically, the set of $d^{t h}$ perfect powers, or the set of prime numbers are strongly ergodic (according to the result of Vinogradov for $t$ irrational mod. $2 \pi$, and to the Dirichlet's arithmetic progression theorem for $t$ rational mod. $2 \pi$ ). The third name comes from $\mathrm{H}$. Weyl's classical criterion for the equidistribution of a real sequence mod. $2 \pi$.

The relationship between these notions comes from:

Theorem 1.9 (F. Lust-Piquard [42]) Let $\Lambda \subseteq[1,+\infty[$ be a set of positive integers. Then:

(a) If $\Lambda$ is strongly ergodic, $\mathcal{C}_{\Lambda}$ contains $c_{0}$.

(b) More generally, if $\Lambda$ is strongly ergodic and $D \subseteq \Lambda$ has a positive upper density with respect to $\Lambda$, then $\mathcal{C}_{D}$ contains $c_{0}$ as well.

Here "positive upper density" means that:

$$
\varlimsup_{N \rightarrow+\infty} \frac{|D \cap[1, N]|}{|\Lambda \cap[1, N]|}>0 .
$$

Part (b) will be useful to us in the last theorem of Section 2 ,

See [42] for the proof of this theorem. The underlying idea for (a) is that if $A_{N}(t) \rightarrow l_{\Lambda}(t)$ for every $t \in \mathbb{T}, l_{\Lambda}$ defines an element of the biorthogonal $\mathcal{C}_{\Lambda}^{\perp \perp}$, and the condition $l_{\Lambda} \in c_{0}(\mathbb{T})$ implies that it is the sum of a weakly unconditionally Cauchy series of continuous functions. By using a perturbation 
argument due to A. Pełczyński (see [55], lemma 15.7, p. 446) and the classical Bessaga-Pełczyński theorem, one obtains that $\mathcal{C}_{\Lambda}$ contains $c_{0}$.

This theorem allowed its author to prove that $\mathcal{C}_{\Lambda}$ contains $c_{0}$ when $\Lambda=$ $\left\{1,2^{d}, 3^{d}, \ldots\right\}$ is the set of the $d^{t h}$ perfect powers, and when $\Lambda=\{2,3,5,7, \ldots\}$ is the set of the prime numbers. On the other hand, K. I. Oskolkov (44]; see also [1]) showed that the set of the $d^{\text {th }}$ powers is not a UC-set, and J. Fournier and L. Pigno ([21], Th. 4) proved that the set of prime numbers is not a UC-set either. This could be taken as an indication that containing $c_{0}$ is an obstruction to being UC. As we shall see in the next section, this is far from being the case: there do exist sets $\Lambda$ which are $\mathrm{UC}$ and for which $\mathcal{C}_{\Lambda}$ contains $c_{0}$.

The last ingredient we require is a random procedure to produce ergodic sets.

Let $\left(\varepsilon_{k}\right)_{k \geq 1}$ be a sequence of independent $0-1$ valued random variables, called "selectors" according to the terminology coined by J. Bourgain. To those selectors is associated a random set $\Lambda$ of positive integers

$$
\Lambda=\Lambda(\omega)=\left\{k \geq 1 ; \varepsilon_{k}(\omega)=1\right\} .
$$

Theorem 1.10 (J. Bourgain [10], Prop. 8.2) Let $\varepsilon_{1}, \ldots, \varepsilon_{N}, \ldots$ be selectors of respective expectations $\delta_{1}, \ldots, \delta_{N}, \ldots$ and assume that $\sigma_{N} / \log N \rightarrow+\infty$, where $\sigma_{N}=\delta_{1}+\cdots+\delta_{N}$ (which is in particular the case when $k \delta_{k} \rightarrow+\infty$ ), and that $\left(\delta_{n}\right)_{n}$ decreases. Then the set $\Lambda=\Lambda(\omega)$ is almost surely uniformly distributed. In particular, it is almost surely strongly ergodic.

\section{Main results}

In this section, we will always consider selectors $\varepsilon_{n}, n \geq 1$, with mean $\delta_{n}=\alpha_{n} / n$, with $\left(\alpha_{n}\right)_{n}$ tending to infinity and $\left(\delta_{n}\right)_{n}$ decreasing.

Moreover, except in the last theorem of this section, we will assume that $\left(\alpha_{n}\right)_{n}$ is increasing.

If $\Lambda=\Lambda(\omega)=\left\{n \geq 1 ; \varepsilon_{n}(\omega)=1\right\}$ is the corresponding random set of integers, $\Lambda$ is almost surely uniformly distributed by Bourgain's theorem. Moreover, it also has the nice almost sure property of being asymptotically independent; more precisely, there exists an increasing sequence $\left(M_{n}\right)_{n}$ of positive integers such that $\Lambda \cap\left[M_{n},+\infty[\right.$ is both large and without relations of length $\leq n$. A subset $B$ of $\Lambda \cap\left[M_{n},+\infty[\right.$ with $n$ elements is then automatically quasi-independent, and this allows us to use Propositions 1.1, 1.2, 1.6 to show that $\Lambda$ has good additional properties: UC, $p$-Sidon, etc... To obtain this asymptotic quasiindependence, the following half-combinatorial, half-probabilistic lemma plays a crucial role.

Recall that $\sigma_{n}=\delta_{1}+\cdots+\delta_{n}$.

Lemma 2.1 Let $s \geq 2$ and $M$ be integers. Set

$$
\Omega_{s}(M)=\{\omega \in \Omega ; \Lambda(\omega) \cap[M,+\infty[\text { contains at least a relation of length } s\} .
$$


Then:

$$
\mathbb{P}\left(\Omega_{s}(M)\right) \leq \frac{s 2^{s-2}}{(s-2) !} \sum_{j>M} \delta_{j}^{2} \sigma_{j}^{s-2} \leq \frac{(4 \mathrm{e})^{s}}{s^{s}} \sum_{j>M} \delta_{j}^{2} \sigma_{j}^{s-2} .
$$

The important fact in this lemma is the presence of the exponent 2 in the factor $\delta_{j}^{2}$ and of the factorial in the denominator.

Proof. We thank the referee for suggesting the following proof.

We have $\Omega_{s}(M)=\bigcup_{l \geq M+s-1} \Delta_{l}$, where $\Delta_{l}=\Delta(l, M, s)$ is defined by:

$$
\Delta_{l}=\{\omega ; \Lambda(\omega) \cap[M,+\infty[
$$

contains at least a relation of length $s$, with largest term $l\}$.

In other words, $\omega \in \Delta_{l}$ if and only if $\Lambda(\omega) \cap[M,+\infty$ [ has at least a relation of length $s$ which contains $l$ and which is contained in $\{M, \ldots, l\}$.

We clearly have:

$$
\Delta_{l} \subseteq \bigcup_{\left(i_{1}, \ldots, i_{s-1}\right)} \Delta\left(l, i_{1}, \ldots, i_{s-1}\right)
$$

where

$$
\Delta\left(l, i_{1}, \ldots, i_{s-1}\right)=\left\{\omega ; \varepsilon_{i_{1}}(\omega)=\cdots=\varepsilon_{i_{s-1}}(\omega)=\varepsilon_{l}(\omega)=1\right\},
$$

and where $\left(i_{1}, \ldots, i_{s-1}\right)$ runs over the $(s-1)$-tuples of integers such that:

(*) $M \leq i_{1}<\cdots<i_{s-1}<l$,

(**) $\theta_{1} i_{1}+\theta_{2} i_{2}+\cdots+\theta_{s-1} i_{s-1}+\theta_{s} l=0, \quad \theta_{1}, \ldots, \theta_{s} \in\{-1,+1\}$.

Observe that $\delta_{i_{s-1}} \leq(s-1) \delta_{l}$ for such $(s-1)$-tuples. In fact, it follows from $(* *)$ that $l \leq i_{1}+\cdots+i_{s-1} \leq(s-1) i_{s-1}$, so

$$
\delta_{i_{s-1}}=\frac{\alpha_{i_{s-1}}}{i_{s-1}} \leq \frac{\alpha_{l}}{i_{s-1}}=\frac{\alpha_{l}}{l} \frac{l}{i_{s-1}} \leq(s-1) \delta_{l} .
$$

Observe also that, when $i_{1}, \ldots, i_{s-2}$ are fixed, $i_{s-1}= \pm l \pm i_{s-2} \pm \cdots \pm i_{1}$ can take at most $2^{s-1}$ values, so that

$$
\begin{aligned}
\mathbb{P}\left(\Delta_{l}\right) & \leq \sum \mathbb{P}\left(\Delta\left(l, i_{1}, \ldots, i_{s-1}\right)\right)=\sum \delta_{i_{1}} \ldots \delta_{i_{s-1}} \delta_{l} \\
& \leq(s-1) 2^{s-1} \delta_{l}^{2} \sum_{M \leq i_{1}<\cdots<i_{s-2} \leq l-1} \delta_{i_{1}} \ldots \delta_{i_{s-2}} \\
& \leq(s-1) 2^{s-1} \delta_{l}^{2} \frac{\left(\delta_{M}+\cdots+\delta_{l-1}\right)^{s-2}}{(s-2) !}
\end{aligned}
$$

by the multinomial formula.

Therefore, noting that $s \geq 2$, we have:

$$
\mathbb{P}\left(\Omega_{s}(M)\right) \leq \sum_{l \geq M+s-1}(s-1) 2^{s-1} \delta_{l}^{2} \frac{\sigma_{l-1}^{2}}{(s-2) !} \leq \sum_{j>M} \frac{(s-1) 2^{s-1}}{(s-2) !} \delta_{j}^{2} \sigma_{j}^{s-2} .
$$


The following theorem is the main result of the paper. It states that subsets of integers can, in several ways, be very close to Sidon sets, but in the same time be rather large.

Theorem 2.2 There exist sets $\Lambda$ of integers which are:

(1) $p$-Sidon for all $p>1, \Lambda(q)$ for all $q<+\infty$, and CUC, but which are also

(2) uniformly distributed; in particular, they are dense in the Bohr group, and $\mathcal{C}_{\Lambda}$ contains $c_{0}$, so $\Lambda$ is not a Rosenthal set.

Proof. We use selectors $\varepsilon_{k}$ of mean

$$
\delta_{k}=c \frac{\log \log k}{k} \quad(k \geq 3),
$$

where $c$ is a constant to be specified latter. Since this constant plays no role in the beginning of the proof, for convenience, we first assume that $c=1$.

The last assertion follows at once from Bourgain's and Lust-Piquard's theorems. The rest of the proof depends on the following lemma, where we set $\Lambda_{n}=\Lambda \cap[1, n]$ and $\Lambda_{n}^{\prime}=\Lambda \cap\left[M_{n}, M_{n+1}[\right.$.

Lemma 2.3 If $M_{n}=n^{n}$, one has the following properties, where $C_{0}$ denotes a numerical constant:

(1) $\sum_{n \geq 3} \mathbb{P}\left(\Omega_{n}\left(M_{n}\right)\right)<+\infty$.

(2) Almost surely $\left|\Lambda_{M_{n}}\right| \leq C_{0} n(\log n)^{2}$ for $n$ large enough.

(3) Almost surely $\left|\Lambda_{n}^{\prime}\right| \leq C_{0}(\log n)^{2}$ for $n$ large enough.

Proof of Lemma 2.3. Note first that

$\sigma_{n}=\sum_{3 \leq k \leq n} \frac{\log \log k}{k} \leq(\log \log n) \sum_{3 \leq k \leq n} \frac{1}{k} \leq(\log \log n) \int_{1}^{n} \frac{d t}{t}=\log n \log \log n$.

Now, take $n \geq 64$, and use Lemma 2.1 to obtain (setting $C=4 \mathrm{e}$ ):

$$
\begin{aligned}
\mathbb{P}\left(\Omega_{n}\left(M_{n}\right)\right) & \leq \frac{C^{n}}{n^{n}} \sum_{j>M_{n}}\left(\frac{\log \log j}{j}\right)^{2}(\log j \log \log j)^{n-2} \\
& \leq \frac{C^{n}}{n^{n}} \sum_{j>M_{n}} \frac{(\log j \log \log j)^{n}}{j^{2}} .
\end{aligned}
$$

But, for fixed $n$, the function

$$
\frac{u}{v}=\frac{(\log x \cdot \log \log x)^{n}}{x^{2}}
$$

decreases on $\left[M_{n},+\infty\left[\right.\right.$. Indeed, we have to check that $u^{\prime}(x) v(x) \leq u(x) v^{\prime}(x)$ on this interval, i.e. that

$$
n x(1+\log \log x)(\log x \log \log x)^{n-1} \leq 2 x(\log x \log \log x)^{n}
$$


or, equivalently, that

$$
n(1+\log \log x) \leq 2 \log x \log \log x .
$$

Now, if $x \geq n^{n}$, we see that

$$
n(1+\log \log x) \leq 2 n \log \log x \leq 2 n \log n \log \log x \leq 2 \log x \log \log x .
$$

Therefore,

$$
\mathbb{P}\left(\Omega_{n}\left(M_{n}\right)\right) \leq \frac{C^{n}}{n^{n}} \int_{M_{n}}^{+\infty} \frac{(\log t \log \log t)^{n}}{t^{2}} d t
$$

Setting

$$
f_{n}(t)=\frac{(\log t \log \log t)^{n}}{t^{2}} \quad \text { and } \quad I_{n}=\int_{M_{n}}^{+\infty} f_{n}(t) d t
$$

we have, by summation by parts:

$$
I_{n}=\frac{\left(\log M_{n} \log \log M_{n}\right)^{n}}{M_{n}}+\int_{M_{n}}^{+\infty} n f_{n}(t)\left(\frac{1}{\log t}+\frac{1}{\log t \log \log t}\right) d t .
$$

Since the function in the integrand is less than

$$
\frac{2 n}{\log t} f_{n}(t) \leq \frac{2 n}{n \log n} f_{n}(t) \leq \frac{1}{2} f_{n}(t)
$$

(recall that $n \geq 64$ ), this gives:

$$
I_{n} \leq \frac{\left(\log M_{n} \log \log M_{n}\right)^{n}}{M_{n}}+\frac{1}{2} I_{n},
$$

so:

$$
\begin{aligned}
\mathbb{P}\left(\Omega_{n}\left(M_{n}\right)\right) \leq \frac{C^{n}}{n^{n}} I_{n} & \leq 2 \frac{C^{n}}{n^{n}} \frac{\left(\log M_{n} \log \log M_{n}\right)^{n}}{M_{n}} \\
& \leq 2 \frac{C^{n}}{n^{n}} \frac{(n \log n \cdot 2 \log n)^{n}}{M_{n}}=2 \frac{(2 C)^{n}(\log n)^{2 n}}{n^{n}},
\end{aligned}
$$

which proves (1).

To prove (2), first note that

$$
\sigma_{n} \geq \sum_{16 \leq k \leq n} \frac{1}{k} \geq \frac{1}{2} \log n \quad \text { for } n \geq 256 .
$$

Now, using Lemma 1.3 with $X_{k}=\varepsilon_{k}-\delta_{k}$, we obtain:

$$
\begin{aligned}
\mathbb{P}\left(|| \Lambda_{n}\left|-\sigma_{n}\right| \geq \frac{\sigma_{n}}{2}\right) & =\mathbb{P}\left(\left|\sum_{3 \leq k \leq n} X_{k}\right| \geq \frac{\sigma_{n}}{2}\right) \\
& \leq 4 \exp \left(-\frac{\sigma_{n}}{32}\right) \leq 4 \exp \left(-\frac{\log n}{64}\right) .
\end{aligned}
$$


In particular, since $M_{n}=n^{n}$,

$$
\mathbb{P}\left(|| \Lambda_{M_{n}}\left|-\sigma_{M_{n}}\right| \geq \frac{\sigma_{M_{n}}}{2}\right) \leq 4 \exp \left(-\frac{n \log n}{64}\right),
$$

and the Borel-Cantelli lemma shows that, almost surely,

$$
|| \Lambda_{M_{n}}\left|-\sigma_{M_{n}}\right|<\sigma_{M_{n}} / 2
$$

for $n$ large enough (depending on $\omega$ ). Thus:

$$
\left|\Lambda_{M_{n}}\right| \leq 2 \sigma_{M_{n}} \leq 2 \log M_{n} \log \log M_{n} \leq C_{0} n(\log n)^{2},
$$

for some numerical constant $C_{0}$, and this gives (2).

The proof of (3) goes the same way. Set:

$$
\sigma_{n}^{\prime}=\sum_{M_{n} \leq k<M_{n+1}} \frac{\log \log k}{k}
$$

and observe that (here, and in the remainder of the paper, the sign $\sim$ between two functions will mean that these two functions are equivalent up to a constant factor):

$$
\sigma_{n}^{\prime} \sim \log n \sum_{M_{n} \leq k<M_{n+1}} \frac{1}{k} \sim \log n \log \frac{M_{n+1}}{M_{n}} \sim(\log n)^{2},
$$

so that:

$$
C_{0}^{-1}(\log n)^{2} \leq \sigma_{n}^{\prime} \leq C_{0}(\log n)^{2}
$$

for some numerical constant $C_{0}$.

Then, using again Lemma 1.3, we get:

$$
\mathbb{P}\left(|| \Lambda_{n}^{\prime}\left|-\sigma_{n}^{\prime}\right| \geq \sigma_{n}^{\prime} / 2\right) \leq 4 \exp \left(-\frac{\sigma_{n}^{\prime}}{32}\right) \leq 4 \exp \left(-\frac{(\log n)^{2}}{32 C_{0}}\right) ;
$$

so the Borel-Cantelli lemma shows that, almost surely,

$$
\left|\Lambda_{n}^{\prime}\right| \leq 2 \sigma_{n}^{\prime} \leq 2 C_{0}(\log n)^{2}
$$

for $n$ large enough, which gives (3), provided we enlarge $C_{0}$, and completes the proof of Lemma 2.3.

We now conclude the proof of Theorem 2.2.

We first choose the constant $c$ in order that, not only $\sum_{n \geq 3} \mathbb{P}\left(\Omega_{n}\left(M_{n}\right)\right)<$ $+\infty$, but $\sum_{n>3} \mathbb{P}\left(\Omega_{n}\left(M_{n}\right)\right)<1$. So, using Lemma 2.3, we can find $\Omega_{0} \subseteq \Omega$ such that $\mathbb{P}\left(\Omega_{0}\right)>0$ and with the property:

$$
\begin{aligned}
& \text { If } \omega \in \Omega_{0} \text {, then } \omega \notin \bigcup_{n \geq 3} \Omega_{n}\left(M_{n}\right) \text {. There exists } n_{0}=n_{0}(\omega) \text { such that } \\
& \left|\Lambda_{2 M_{n}}\right| \leq C_{0} n(\log n)^{2} \text { and }\left|\Lambda_{n}^{\prime}\right| \leq C_{0}(\log n)^{2} \leq n \text { for } n>n_{0} .
\end{aligned}
$$


Indeed, an inspection of the proof of Lemma 2.3 shows that we also have, almost surely, $\left|\Lambda_{2 M_{n}}\right| \leq 2 \sigma_{2 M_{n}} \leq C_{0} n(\log n)^{2}$ for $n$ large enough, and this gives (2.1). We have the following consequences, where $\omega \in \Omega_{0}$, and $\Lambda=\Lambda(\omega)$ :

$$
\Lambda \cap\left[M_{n},+\infty[\text { contains no relation of length } \leq n .\right.
$$

For, if $\Lambda \cap\left[M_{n},+\infty[\right.$ were to contain a relation $R$ of support $S$ with $|S|=s \leq n$, then necessarily $s \geq 3, S \subseteq \Lambda \cap\left[M_{s},+\infty\left[\right.\right.$ and $\omega \in \Omega_{s}\left(M_{s}\right)$, which is not the case by (2.1). Now, (2.1) and (2.2) imply that, for $n>n_{0}, \Lambda_{n}^{\prime}$ is quasi-independent, and so is a Sidon set with bounded constant. So, we get that $\Lambda$ is CUC and $\Lambda(q)$ for all $q<+\infty$ using Propositions 1.1 and 1.2, provided we notice that:

$$
\frac{M_{n+1}}{M_{n}}=\frac{(n+1)^{n+1}}{n^{n}} \geq n+1 \geq 2 .
$$

To end the proof, we first show that $\Lambda$ is $p$-Rider, for every $p>1$, and then, using Proposition 1.6 (c), prove that it is $q$-Sidon for every $q>1$.

So, fix $p \in] 1,2[$, set $\varepsilon=2 / p-1 \in] 0,1$ [, and take $\omega \in \Omega_{0}$ and $n_{1}=n_{1}(\varepsilon, \omega) \geq$ $2 n_{0}(\omega)$ such that $C_{0} n(\log n)^{2} \leq n^{1 / \varepsilon} / 2$ and $n^{1 / \varepsilon} / 2 \geq n$ for $n \geq n_{1}$.

Let $A \subseteq \Lambda$ be a finite subset, with $|A|^{\varepsilon}>n_{1}$. Set $n=\left[|A|^{\varepsilon}\right]$, where [ ] stands for integer part, so that $n \geq n_{1}$ and $|A| \geq n^{1 / \varepsilon}$. Observe that:

$$
\begin{aligned}
\mid A \cap\left[M_{n},+\infty[\mid\right. & \geq|A|-\left|A \cap\left[1, M_{n}\right]\right| \geq|A|-\left|\Lambda_{M_{n}}\right| \\
& \geq n^{1 / \varepsilon}-C_{0} n(\log n)^{2} \geq n^{1 / \varepsilon} / 2 \geq n,
\end{aligned}
$$

and select $B \subseteq A \cap\left[M_{n},+\infty[\right.$ with $|B|=n$. It follows from (2.2) that $B$ is quasi-independent, and $|B|=n \geq \frac{1}{2}|A|^{\varepsilon}$.

If now $A$ is a subset of $\Lambda$ with $1 \leq|A| \leq n_{1}$, simply take for $B$ a singleton from $A$. Then $B$ is quasi-independent, and $|B|=1 \geq n_{1}^{-1}|A|^{\varepsilon}$.

The criterion of Proposition 1.6 (a) is verified with $\delta=n_{1}^{-1}$. Therefore $\Lambda$ is $p$-Rider.

We shall verify that we are in position to apply part (b) of Proposition 1.6

Take $p \in] 1,2\left[\right.$ and $1 / p<\varepsilon<1$. Take $\omega \in \Omega_{0}$ and $n_{1}=n_{1}(\varepsilon, \omega) \geq 2 n_{0}(\omega)$ such that $C_{0} n(\log n)^{2} \leq n^{1 / \varepsilon} / 2$ and $n^{1 / \varepsilon} / 2 \geq n$ for $n \geq n_{1}$.

Let $A \subseteq \Lambda$ be a finite subset with $|A|^{\varepsilon}>n_{1}$. Set $n=\left[|A|^{\varepsilon}\right]$, where [ ] stands for integer part, so that $n \geq n_{1}$ and $|A| \geq n^{1 / \varepsilon}$. Observe that:

$$
\begin{aligned}
\mid A \cap\left[2 M_{n},+\infty[\mid\right. & \geq|A|-\left|A \cap\left[1,2 M_{n}\right]\right| \geq|A|-\left|\Lambda_{2 M_{n}}\right| \\
& \geq n^{1 / \varepsilon}-C_{0} n(\log n)^{2} \geq n^{1 / \varepsilon} / 2 \geq n
\end{aligned}
$$

in view of (2.1). We can thus select $B \subseteq A \cap\left[2 M_{n},+\infty[\right.$ with $|B|=n-1$ and have:

If $k \in \Lambda \cap\left[M_{n},+\infty[\right.$, then $B \cup\{k\}$ is quasi-independent.

Indeed, $B \cup\{k\}$ is a set of cardinality less than $n$ contained in $\Lambda \cap\left[M_{n},+\infty\right.$, and is automatically quasi-independent, from (2.2).

We show now that $B$ is 8 -pseudo-complemented in $\Lambda$. 
Put $\nu=\delta_{0}-V_{M_{n}}$, where $\delta_{0}$ is the Dirac point mass at 0 , and $V_{M_{n}}$ the de la Vallée-Poussin kernel of order $M_{n}$. Consider the Riesz product $R=$ $\prod_{k \in B}\left(1+\mathfrak{R e} e_{k}\right)$, and set $\mu=2 \nu * R$. We claim that:

$$
\|\mu\| \leq 8 ; \quad \widehat{\mu} \geq 1 \quad \text { on } B ; \quad \widehat{\mu}=0 \quad \text { on } \Lambda \backslash B .
$$

Indeed, $\|\nu\| \leq 4$ and $B$ is quasi-independent, so the Riesz product $R$ verifies $\|R\|=\widehat{R}(0)=1$. Therefore $\|\mu\| \leq 8$.

Take $l \in B$. Then $l>2 M_{n}$ and $\widehat{\nu}(l)=1$. As $\widehat{R}(l) \geq 1 / 2$, we have $\widehat{\mu}(l) \geq 1$.

If $A \subseteq \Lambda$ and $|A|^{\varepsilon} \leq n_{1}$, any singleton $B$ of $A$ is quasi-independent, 1complemented in $\Lambda$, and $|B| \geq n_{1}^{-1}|A|^{\varepsilon}$.

We have thus verified the hypothesis of part (b) of Proposition 1.6, and so $\Lambda$ is $q$-Sidon for any $q>1 / \varepsilon$. In particular, it is $p$-Sidon, and this ends the proof of Theorem 2.2.

Remark 1. The proof shows that we can actually extract from $A$, for every $\alpha>0$, a quasi-independent set $B$ such that $|B| \geq \delta|A| /(\log |A|)^{2+\alpha}$. Moreover, a slight modification leads to sets even closer to Sidon sets.

Proposition 2.4 Let $\alpha>1$ and $\varphi_{\alpha}$ be the Orlicz function $x \mapsto x(\log (1+x))^{\alpha}$. Then, there exists a set $\Lambda$ as in Theorem 2.2, and moreover such that $\widehat{f} \in \ell_{\varphi_{\alpha}, \infty}$ for every $f \in \mathcal{C}_{\Lambda}$.

Recall that $\ell_{\varphi_{\alpha}, \infty}$ is the weak Orlicz-Lorentz space of sequences $\left(a_{n}\right)_{n}$ such that $\sup _{n} \varphi_{\alpha}^{-1}(n) a_{n}^{*}<+\infty$, where $\left(a_{n}^{*}\right)_{n}$ is the non-increasing rearrangement of $\left(\left|a_{n}\right|\right)_{n}$. Therefore, another way to phrase the proposition is, setting $a_{n}=\widehat{f}(n)$ :

$$
a_{n}^{*} \leq C_{\alpha}\|f\|_{\infty}(\log n)^{\alpha} / n \text { for every } f \in \mathcal{C}_{\Lambda} .
$$

The proof just consists in changing $M_{n}$. We take $M_{n}=\left[e^{n(\log \log n)^{2}}\right]$, where [ ] stands for the integer part. We still have $\sum_{n} \mathbb{P}\left(\Omega_{n}\left(M_{n}\right)\right)<+\infty$, since

$$
\mathbb{P}\left(\Omega_{n}\left(M_{n}\right)\right) \leq \frac{2 C^{n}}{n^{n}} \frac{\left(\log M_{n} \log \log M_{n}\right)^{n}}{M_{n}} \leq \exp \left(-n(\log \log n)^{2} / 2\right)
$$

for $n$ large enough. Arguing as previously, we get for every finite subset $A$ of $\Lambda$, a quasi-independent subset $B$ of $A$ such that $|B| \geq \delta|A| /(\log |A|)^{\alpha}$, and such that $\mathcal{C}_{B}$ is uniformly pseudo-complemented in $\mathcal{C}_{A}$. As in the proof of Proposition 1.6. we obtain

$$
|\{|\widehat{f}|>t\}| \leq C \varphi_{\alpha}\left(\frac{\|f\|_{\infty}}{t}\right),
$$

which gives the result (arguing as in [34] for instance).

We cannot eliminate a logarithmic factor, and replace $\alpha>1$ by $\alpha>0$ because, due to Bourgain's criterion, we have to assume that $\sigma_{n} / \log n$ goes to 
infinity in order that $\mathcal{C}_{\Lambda}$ contains $c_{0}$. However, for each $\alpha>0$, there do exist non-Sidon sets $\Lambda$ for which $\widehat{f} \in \ell_{\varphi_{\alpha}}$ when $f \in \mathcal{C}_{\Lambda}$ (as can be seen from [5], p. $69)$.

The set $\Lambda$ is, in some sense, very close to be Sidon, whereas $\mathcal{C}_{\Lambda}$ contains $c_{0}$. However, it cannot be too close without being Sidon because if $\widehat{f} \in \ell_{1, \infty}$, the Lorentz space weak- $\ell_{1}$, for every $f \in \mathcal{C}_{\Lambda}$, then $\Lambda$ is Sidon. In fact, this condition implies an inequality of the type:

$$
|\{|\widehat{f}| \geq t\}| \leq \frac{C}{t}\|f\|_{\infty}
$$

for every $f \in \mathcal{C}_{\Lambda}$. Let now $A$ be a finite subset of $\Lambda$, and $f=\sum_{n \in A} e_{n}$ and $f_{\omega}=\sum_{n \in A} r_{n}(\omega) e_{n}$, where $r_{n}, n \geq 1$ are the Rademacher functions. Then, inequality $((*))$ applied with $t=1$ gives $\left\|f_{\omega}\right\|_{\infty} \geq(1 / C)|A|$. Integrating in $\omega$ gives $\llbracket f \rrbracket \geq(1 / C)|A|$, from which follows, by a result of $\mathrm{G}$. Pisier (48], Théorème $2.3(\mathrm{vi}))$, that $\Lambda$ is a Sidon set.

Remark 2. If one takes selectors of mean $\delta_{n}$ such that $n \delta_{n}$ is bounded, the corresponding random set $\Lambda(\omega)$ is almost surely a Sidon set. This is a well-known result of Y. Katznelson and P. Malliavin ([28], or [27]), and Lemma 2.1 gives another proof of this fact. It suffices to take $M_{n}=A^{n}$, where $A$ is a given integer, large enough to have $\sum_{n=1}^{+\infty} \mathbb{P}\left(\Omega_{n}\left(M_{n}\right)\right)<1$. Then, with positive probability $\Lambda(\omega) \cap\left[M_{n},+\infty\left[\right.\right.$ contains no relation of length $\leq n$, whereas $\left|\Lambda(\omega) \cap\left[1, M_{n}\right]\right| \leq$ $C n$. Hence, for every finite subset $A$ of $\Lambda(\omega)$, we can find a quasi-independent subset $B \subseteq A$ such that $|B| \geq \delta|A|$, for some fixed $\delta=\delta(\omega)$. It follows from Pisier's characterization ([48], Th. 2.3 (iv)) that, with positive probability, and hence almost surely by Kolmogorov's $0-1$ law, $\Lambda(\omega)$ is a Sidon set.

As is now well-known, Sidon sets are characterized by various properties (successively weaker) of the Banach space $\mathcal{C}_{\Lambda}: \Lambda$ is a Sidon set iff $\mathcal{C}_{\Lambda}$ is isomorphic to $\ell_{1}([58])$, iff $\mathcal{C}_{\Lambda}$ has cotype 2 ([31], Th. 3.1, [46]), and iff $\mathcal{C}_{\Lambda}$ has a finite cotype (13]). This later property can be expressed by saying that $\mathcal{C}_{\Lambda}$ does not contain $\ell_{\infty}^{n}$ uniformly. So, deterministically, one has the dichotomy:

(a) either $\Lambda$ is a Sidon set, and so $\mathcal{C}_{\Lambda}$ is isomorphic to $\ell_{1}$;

(b) or $\mathcal{C}_{\Lambda}$ contains $\ell_{\infty}^{n}$ uniformly.

The probabilistic dichotomy is stronger: taking selectors of mean $\delta_{1}, \delta_{2}, \ldots$, with $\left(\delta_{n}\right)_{n}$ decreasing, one has:

(a) either almost surely $\Lambda$ is a Sidon set (if $n \delta_{n}$ is bounded);

(b) or almost surely $\mathcal{C}_{\Lambda}$ contains $c_{0}$ (if $n \delta_{n}$ is not bounded), and $\Lambda$ is even uniformly distributed.

Y. Katznelson (27]) already noticed such a "dichotomy": he showed that (under a different choice of selectors from ours) either almost surely $\Lambda$ is a Sidon set, or almost surely $\Lambda$ is dense in the Bohr group. However, this is perhaps not a true dichotomy since it is a well-known open problem whether there can exist Sidon sets dense in the Bohr group (see [15], question 2, p. 14; 
it is stated for the Bohr group of $\mathbb{R}$, but also makes sense for the Bohr group of $\mathbb{Z})$.

The dichotomy stated here strengthens Katznelson's result since every uniformly distributed set is dense in the Bohr group (see [6], Theorem 1); indeed, saying that $\Lambda=\left\{\lambda_{1}, \lambda_{2}, \ldots\right\}$ is uniformly distributed means that the measures $\mu_{N}=1 / N \sum_{n=1}^{+\infty} \delta_{\lambda_{n}}\left(\delta_{\lambda_{n}}\right.$ is there the Dirac measure at the point $\left.\lambda_{n}\right)$ converge weak-star to the Haar measure $\mu$ of the Bohr group $b \mathbb{Z}$; but these measures are carried by $\Lambda$, so the closed support of $\mu$ is contained in the Bohr closure of $\Lambda$; since the Haar measure is continuous, we get that this closure is the whole Bohr group.

Remark 3. The random sets $\Lambda$ that we construct have an asymptotical quasiindependence: $\Lambda \cap\left[M_{n},+\infty[\right.$ contains no relation of length $\leq n$. This is reminiscent of the following result of J. Bourgain ([7]): if $\Lambda$ is a Sidon set and $n \in \mathbb{N}^{*}$, there exists $l_{n}=l(\Lambda, n)$ such that $\Lambda$ can be decomposed in $l_{n}$ sets $\Lambda_{1}, \ldots, \Lambda_{l_{n}}$, each of which contains no relation of length $\leq n$.

We now investigate what happens when we let $p$ increase away from 1 .We get several different results, and $p=4 / 3$ seems to play a special role.

We first state two very similar results.

Theorem 2.5 For every $1<p<4 / 3$, there exists a set $\Lambda$ of integers which is:

(1) uniformly distributed (so $\Lambda$ is dense in the Bohr group, $\mathcal{C}_{\Lambda}$ contains $c_{0}$, and $\Lambda$ is not a Rosenthal set), and which is:

(2) $\Lambda(q)$ for all $q<+\infty$, a CUC-set, and moreover is:

(a) $p$-Rider, but not $q$-Rider for $q<p$

(b) $q$-Sidon for all $q>p /(2-p)$.

Theorem 2.6 Same as Theorem 2.5, except that, instead of (a), $\Lambda$ is:

(a') $q$-Rider for every $q>p$, but is not $p$-Rider.

Remark. After this paper was completed, P. Lefèvre and the third-named author proved ([36]) that every $p$-Rider set with $p<4 / 3$ is a $q$-Sidon set, for all $q>p /(2-p)$. A weaker, unpublished, result, due to J. Bourgain, is quoted in [15], p. 41. Hence condition (b) always follows from condition (a), and is not specific to the construction. We do not know whether this gap between $p$ and $p /(2-p)$ follows only from technical reasons. For $p>1$, whether every $p$-Rider set is actually $p$-Sidon is an open question.

In Theorem 2.5 we obtain sets which are $p$-Rider but not $q$-Rider for $q<p$. We do not know if these sets are $p$-Sidon, so exactly $p$-Sidon, in the terminology of R. Blei. He constructed such sets using fractional products ([3], 4]). We may call the sets in Theorem 2.5 "exactly p-Rider sets". The sets appearing in Theorem 2.6 are of a different kind. We may call them "exactly $p^{+}$-Rider sets". Such sets were also obtained in [3], Corol. $1.7 \mathrm{~d}$ ), where they were called "exactly non- $p$-Sidon", and were called "asymptotic $p$-Sidon" in [5]. 
Proof. It is similar to that of Theorem 2.2, so we shall be very sketchy.

Let $\alpha=2(p-1) /(2-p) \in] 0,1[$.

For Theorem 2.5, we use selectors $\varepsilon_{k}$ of mean

$$
\delta_{k}=c \frac{(\log k)^{\alpha}}{k(\log \log k)^{\alpha+1}} \quad \text { for } k \geq 4 .
$$

As in Lemma 2.3, we have, with $M_{n}=n^{n}, \sum_{n>1} \mathbb{P}\left(\Omega_{n}\left(M_{n}\right)\right)<+\infty$, and almost surely $C_{0} n^{\alpha+1} \leq\left|\Lambda_{M_{n}}\right| \leq C_{1} n^{\alpha+1}$ and $\left|\Lambda_{n}^{\prime}\right| \leq C_{1} n^{\alpha}$ for $n$ large enough.

For Theorem 2.6. we increase the means $\delta_{k}$ slightly, replacing them by

$$
\delta_{k}=c \frac{(\log k)^{\alpha} \log \log k}{k} .
$$

Remark. In order to prove our theorems, we used selectors with various means. They are smaller in Theorem 2.2 than in Theorem 2.5 for instance. We remark that selectors $\left(\varepsilon_{k}\right)_{k}$ of mean $\delta_{k}$ with $\delta_{k} \leq \delta_{k}^{\prime}$ may be achieved as the product of two independent sequences of selectors $\left(\varepsilon_{k}^{\prime}\right)_{k}$ and $\left(\varepsilon_{k}^{\prime \prime}\right)_{k}$ of mean $\delta_{k}^{\prime}$ and $\delta_{k}^{\prime \prime}=$ $\delta_{k} / \delta_{k}^{\prime}$. It follows that, for example, the sets in Theorem 2.2 may be constructed inside the respective sets of Theorem 2.5

In Theorem 2.5 the proof that $\Lambda$ was $C U C$ or $\Lambda(q)$ was based on the fact that $\left|\Lambda_{n}^{\prime}\right| \subseteq \Lambda \cap\left[M_{n},+\infty[\right.$ is quasi-independent. For $\alpha \geq 1$ (i.e. $p \geq 4 / 3$ ), we no longer have $\left|\Lambda_{n}^{\prime}\right| \leq n$, and therefore, a priori, must give up these properties. However, we can use another extraction procedure. This procedure was first introduced by J. Bourgain ([8]); later, a clear statement was given in [52], § III.2. Since this last reference is hardly available, we prefer to give a self-contained proof.

The corresponding set $\Lambda(\omega)$ of integers that we shall obtain in this manner satisfies $\mid \Lambda(\omega) \cap\left[2^{n}, 2^{n+1}\left[\mid \sim n \sim \log 2^{n}\right.\right.$, which is the limiting condition of mesh (on arithmetic progressions) for Sidon sets. This size is in some sense the largest possible if we want to obtain a set $\Lambda$ with blocks having a uniformly bounded Sidon constant.

Theorem 2.7 There exists a set $\Lambda$ of integers which is uniformly distributed and contains a subset $E \subseteq \mathbb{N}^{*}$ which is:

(1) 4/3-Rider, and not $q$-Rider for $q<4 / 3$; a CUC-set; a $\Lambda(q)$-set for all $q<+\infty$ (more precisely, for all $q>2$, we have: $\|f\|_{q} \leq C q^{2}\|f\|_{2}$ for all $f \in \mathcal{P}_{E}$, where $C>0$ is a numerical constant), and nevertheless,

(2) has positive upper density in $\Lambda$, so, in particular, $\mathcal{C}_{E}$ contains $c_{0}$, and $E$ is not a Rosenthal set.

Let $A$ be a finite subset of integers. For the proof, it will be convenient to define:

$$
\psi_{A}=\sup _{p \geq 2} \frac{\left\|e_{A}\right\|_{p}}{\sqrt{p}}, \quad \text { where } e_{A}=\sum_{k \in A} e_{k} .
$$

We need the following simple estimate of $\psi_{A}$. 
Lemma 2.8 Let $I=[a+1, a+N]$ be an interval of integers of length $N, N \geq 3$. Then:

$$
\psi_{I} \leq \frac{N}{\sqrt{2 \log N}} .
$$

Proof. For $p \geq 2,\left|e_{I}\right|^{p} \leq N^{p-2}\left|e_{I}\right|^{2}$, so $\int\left|e_{I}\right|^{p} d m \leq N^{p-2} \int\left|e_{I}\right|^{2} d m=N^{p-1}$ and $\left\|e_{I}\right\|_{p} / \sqrt{p} \leq N^{1-1 / p} / \sqrt{p}$. Optimizing gives $p=2 \log N(\geq 2)$, and the lemma.

This estimate is essentially optimal. Indeed, it is well-known that $\psi_{I}$ is uniformly equivalent to $\theta=\left\|e_{I}\right\|_{\Psi}\left(\|\|_{\Psi}\right.$ being the norm associated to the Orlicz function $\left.\Psi(x)=\mathrm{e}^{x^{2}}-1\right)$. But, for some constant $\gamma,\left|e_{I}(t)\right| \geq \gamma N$ for $t$ in an interval $J$ of length $\geq \gamma N^{-1}$ around 0 , so one has:

$$
2 \geq \int_{J} \exp \left(\frac{\left|e_{I}\right|^{2}}{\theta^{2}}\right) d m \geq \gamma N^{-1} \exp \left(\frac{\gamma^{2} N^{2}}{\theta^{2}}\right),
$$

whence $\theta \geq \gamma^{-1} N / \sqrt{\log 2 \gamma^{-1} N}$.

We now use selectors $\varepsilon_{k}$ of mean $\delta_{k}=c n / 2^{n}$ for $2^{n} \leq k<2^{n+1}$, where $c>0$ is a given constant.

Set

$$
I_{n}=\left[2^{n}, 2^{n+1}\left[, n \geq 2 ; \quad \delta_{k}=c \frac{n}{2^{n}} \text { if } k \in I_{n} .\right.\right.
$$

Note that $\left(\delta_{k}\right)_{k}$ decreases, and $\delta_{k}$ is of the form $\alpha_{k} / k$, where $\left(\alpha_{k}\right)_{k}$ goes to $+\infty$.

If $\Lambda=\Lambda(\omega)$ is the corresponding set of integers, it will be convenient to set:

$$
\Lambda_{n}=\Lambda \cap I_{n} ; \quad \sigma_{n}=\mathbb{E}\left|\Lambda_{n}\right|=\sum_{k \in I_{n}} \delta_{k}=c n .
$$

For this proof, the value of $\psi_{I_{n}}$ is somewhat large, and requires $c$ be sufficiently small, say $c \leq 1 / 576$. We prefer to follow another route, which could be useful in other contexts, by choosing also a random set in $I_{n}$ for which the $\psi$ constant is small enough. We make the two random choices at the same time. Namely, we consider $\left(\varepsilon_{n}^{\prime}\right)_{n \geq 1}$, a second sequence of selectors, independent of $\left(\varepsilon_{n}\right)_{n \geq 1}$, with fixed mean $\tau$, and set $\Lambda_{n}^{\prime}(\omega)=\left\{k \in \Lambda_{n}(\omega) ; \varepsilon_{k}^{\prime}(\omega)=1\right\}$. In short:

$$
\Lambda_{n}^{\prime}=\left\{k \in \Lambda_{n} ; \varepsilon_{k}^{\prime}=1\right\} ; \quad \Lambda^{\prime}=\bigcup_{n=1}^{+\infty} \Lambda_{n}^{\prime} .
$$

The following lemma, which is a slight modification of Bourgain's construction in [8, is really the heart of the proof.

Lemma 2.9 Almost surely, for $n$ large enough, one has:

(1) $(c / 2) n \leq\left|\Lambda_{n}\right| \leq(2 c) n$ and $(c \tau / 2) n \leq\left|\Lambda_{n}^{\prime}\right| \leq(2 c \tau) n$

(2) $\Lambda_{n}^{\prime}$ contains at most relations of length $\leq l_{n}$, where $l_{n}=\left[144 c^{2} \tau^{2} n\right]$. 
Proof of Lemma 2.9. We have already seen that:

$$
\mathbb{P}\left(|| \Lambda_{n}\left|-\sigma_{n}\right| \geq \frac{\sigma_{n}}{2}\right) \leq \exp \left(-\frac{\sigma_{n}}{32}\right)=\exp \left(-\frac{c n}{32}\right),
$$

so, by the Borel-Cantelli lemma, $\left|\Lambda_{n}\right|$ is almost surely between $(c / 2) n$ and $(2 c) n$ for $n$ large enough; and this proves the first half of (1). The second half holds for the same reason, since $\Lambda_{n}^{\prime}$ corresponds to selectors $\varepsilon_{k} \varepsilon_{k}^{\prime}$ with mean $(c \tau) n / 2^{n}$ for $k \in I_{n}$.

The proof of (2) is more elaborate.

Fix $n$, and consider the random trigonometric polynomial:

$$
F_{\omega}=\sum_{j=l_{n}+1}^{\left|I_{n}\right|} \sum_{\substack{R \subset I_{n} \\|R|=j}} \prod_{k \in R} \varepsilon_{k}(\omega) \varepsilon_{k}^{\prime}(\omega)\left(e_{k}+e_{-k}\right) .
$$

Set:

$$
N_{n}(\omega)=\int_{\mathbb{T}} F_{\omega}(t) d m(t) .
$$

Expanding $F_{\omega}$, we see that:

$$
\begin{aligned}
F_{\omega}(t) & =\sum_{j=l_{n}+1}^{\left|I_{n}\right|} \sum_{\substack{R \subseteq I_{n} \\
|R|=j}} \sum_{\theta_{k} \in\{-1,+1\}^{R}} \prod_{k \in R} \varepsilon_{k}(\omega) \varepsilon_{k}^{\prime}(\omega) e_{k}^{\theta_{k}}(t) \\
& =\sum_{j=l_{n}+1} \sum_{\substack{R \subseteq I_{n} \\
|R|=j}} \sum_{\theta_{k} \in\{-1,+1\}^{R}} \mathrm{e}^{i t\left(\sum_{k \in R} \theta_{k} k\right) .}
\end{aligned}
$$

The contribution to $N_{n}(\omega)$ of an exponential of this sum is 0 if $\sum_{k \in R} \theta_{k} k \neq 0$, and is 1 if $\sum_{k \in R} \theta_{k} k=0$. Therefore, $N_{n}(\omega)$ is exactly the number of relations of length $>l_{n}$ in $\Lambda_{n}^{\prime}$.

We claim that $N_{n}(\omega)$ is almost surely zero for $n$ large enough. To that effect, we majorize the expectation $J$ of $N_{n}(\omega)$, using Fubini's theorem. Indeed, $J=\int_{\mathbb{T}} H(t) d m(t)$, where:

$$
H(t)=\int_{\Omega} F_{\omega}(t) d \mathbb{P}(\omega)=\sum_{j=l_{n}+1}^{\left|I_{n}\right|} \sum_{\substack{R \subseteq I_{n} \\|R|=j}} \delta^{j} \prod_{k \in R}\left(e_{k}+e_{-k}\right) .
$$

and $\delta=c \tau n / 2^{n}$. Hence:

$$
J=\sum_{j=l_{n}+1}^{\left|I_{n}\right|} \sum_{\substack{R \subseteq I_{n} \\|R|=j}} \delta^{j} \int_{\mathbb{T}} \prod_{k \in R}\left(\left(e_{k}(t)+e_{-k}(t)\right) d m(t) .\right.
$$


At this stage, it is useful to observe that:

$$
\begin{aligned}
\sum_{\substack{R \subseteq I_{n} \\
|R|=j}} \int_{\mathbb{T}} \prod_{k \in R}\left(e_{k}(t)+e_{-k}(t)\right) & d m(t) \\
\leq & \frac{1}{j !} \int_{\mathbb{T}}\left(\sum_{k \in I_{n}}\left(\left(e_{k}(t)+e_{-k}(t)\right)\right)^{j} d m(t) .\right.
\end{aligned}
$$

Indeed, when we expand

$$
\left(\sum_{k \in I_{n}}\left(\left(e_{k}(t)+e_{-k}(t)\right)\right)^{j}\right.
$$

each term $\prod_{k \in R}\left(e_{k}(t)+e_{-k}(t)\right)$ appears $j$ ! times, whereas the other terms on the right hand side of (3) are positive. It now follows from (3) that:

$$
\begin{aligned}
J \leq \sum_{j=l_{n}+1}^{\left|I_{n}\right|} \frac{\delta^{j}}{j !} \int_{\mathbb{T}}\left(\sum_{k \in I_{n}}\right. & \left(\left(e_{k}(t)+e_{-k}(t)\right)\right)^{j} d m(t) \\
\leq & \sum_{j=l_{n}+1}^{\left|I_{n}\right|} \frac{\delta^{j}}{j !} 2^{j}\left\|\sum_{k \in I_{n}} e_{k}\right\|_{j}^{j} \leq \sum_{j=l_{n}+1}^{\left|I_{n}\right|} \frac{2^{j} \delta^{j}}{j !}\left(\psi_{I_{n}} \sqrt{j}\right)^{j} .
\end{aligned}
$$

Since $j ! \geq(j / \mathrm{e})^{j} \geq(j / 3)^{j}$, this gives

$$
J \leq \sum_{j=l_{n}+1}^{+\infty}\left(\frac{6 \delta \psi_{I_{n}}}{\sqrt{j}}\right)^{j} \leq \sum_{j=l_{n}+1}^{+\infty}\left(\frac{6 \delta \psi_{I_{n}}}{\sqrt{l_{n}+1}}\right)^{j} .
$$

Therefore,

$$
J \leq 2^{-l_{n}} \quad \text { if } \quad \frac{6 \delta \psi_{I_{n}}}{\sqrt{l_{n}+1}} \leq \frac{1}{2},
$$

i.e. if $l_{n}+1 \geq 144\left(\delta \psi_{I_{n}}\right)^{2}$. But, it follows from Lemma 2.8 that:

$$
\psi_{I_{n}} \leq \frac{2^{n}}{\sqrt{(2 \log 2) n}} \leq \frac{2^{n}}{\sqrt{n}}
$$

Therefore

$$
144\left(\delta \psi_{I_{n}}\right)^{2} \leq 144\left(c \tau \frac{n}{2^{n}} \cdot \frac{2^{n}}{\sqrt{n}}\right)^{2}=144 c^{2} \tau^{2} n,
$$

and the choice of $l_{n}$ just fits to obtain $J \leq 2^{-l_{n}}$. Of course, we have assumed $n$ large enough to have $l_{n} \geq 1$ in that proof.

Finally, Markov's inequality implies:

$$
\sum_{n \geq 2} \mathbb{P}\left(N_{n} \geq 1\right) \leq \sum_{n \geq 2} \mathbb{E} N_{n} \leq \sum_{n \geq 2} 2^{-l_{n}}<+\infty,
$$


and by the Borel-Cantelli lemma, the integer $N_{n}$ is almost surely zero for $n$ large enough, and that ends the proof of Lemma 2.9.

Now, using Bourgain's Theorem 1.10 and Lemma 2.9, one can find $\Omega_{0} \subseteq \Omega$ with $\mathbb{P}\left(\Omega_{0}\right)=1$ such that for $\omega \in \Omega_{0}$, there exists $n_{0}=n_{0}(\omega)$ such that $\Lambda=\Lambda(\omega)$ and $\Lambda^{\prime}=\Lambda^{\prime}(\omega)$ satisfy:

(4) $\Lambda$ and $\Lambda^{\prime}$ are uniformly distributed

(5) $(c / 2) n \leq\left|\Lambda_{n}\right| \leq(2 c) n$ and $(c \tau / 2) n \leq\left|\Lambda_{n}^{\prime}\right| \leq(2 c \tau) n$ for $n>n_{0}$

(6) $\Lambda_{n}^{\prime}$ contains at most relations of length less than $\leq l_{n}=\left[144 c^{2} \tau^{2} n\right]$ for $n>n_{0}$.

$\Lambda_{n}^{\prime}$ is not quite quasi-independent, so we shall modify it slightly. We adjust once and for all $\tau$, depending on $c$, such that $144 c^{2} \tau^{2} \leq c \tau / 4$ (e.g. taking $c \tau=1 / 576)$, so that $l_{n} \leq c \tau n / 4 \leq\left|\Lambda_{n}^{\prime}\right| / 2$ for $n>n_{0}$, in view of (5). Select then in $\Lambda_{n}^{\prime}$ a relation $R$ with support $S_{n}$ of maximal cardinality. Then $\left|S_{n}\right| \leq l_{n}$ from (6), and $E_{n}=\Lambda_{n}^{\prime} \backslash S_{n}$ is quasi-independent. Moreover:

$$
\left|E_{n}\right|=\left|\Lambda_{n}^{\prime}\right|-\left|S_{n}\right| \geq\left|\Lambda_{n}^{\prime}\right|-l_{n} \geq\left|\Lambda_{n}^{\prime}\right| / 2
$$

for $n>n_{0}$. Hence, if we set $E=\bigcup_{n>n_{0}} E_{n}$, we have $E_{n}=E \cap I_{n}$, and, moreover:

(7) $E$ has positive upper density in $\Lambda$

(note that $\Lambda^{\prime}$ has upper density $\geq \tau / 4$ in $\Lambda$ by (5)),

(8) $E_{n}$ is quasi-independent,

(9) $\left|E_{n}\right| \geq(c \tau / 4) n$,

(10) If $A \subseteq E$ is a finite subset, then $A$ contains a quasi-independent subset $B$ with $|B| \geq(1 / 2)|A|^{1 / 2}$.

The last property is proved in the following way. Set $Z=\left\{n ; A \cap E_{n} \neq \emptyset\right\}$ and $h=|Z|$. We distinguish two cases.

Case 1: there exists $n \in Z$ such that $\left|A \cap E_{n}\right| \geq|A|^{1 / 2}$.

Then, just take $B=A \cap E_{n}$ to have a quasi-independent set $B$ such that $|B| \geq|A|^{1 / 2}$.

Case 2: $\left|A \cap E_{n}\right|<|A|^{1 / 2}$ for any $n \in Z$.

Then $h \geq|A|^{1 / 2}$. Write $Z=\left\{n_{1}<\cdots<n_{h}\right\}$, and pick an integer $m_{j} \in$ $A \cap E_{n_{j}}$ for each $j=1, \ldots, h$. Then $B=\left\{m_{1}, m_{3}, \ldots\right\}=:\left\{\mu_{1}, \mu_{2}, \ldots\right\}$ is quasiindependent because we have $\mu_{j+1} / \mu_{j} \geq 2$. Moreover $|B| \geq h / 2 \geq(1 / 2)|A|^{1 / 2}$.

It is now easy to see that $E$ has the required properties. Indeed, it follows from (4), (7), and from F. Lust-Piquard's Theorem 1.9 that $E$ has a positive upper density in $\Lambda$. That it is CUC follows from (8) and from Proposition 1.2. That it is $\Lambda(q)$ for all $q<+\infty$ follows from (8) and from Proposition [1.1 
The fact that $E$ is $4 / 3$-Rider follows from (a) in Proposition 1.6. Indeed, if $\varepsilon(p)=2 / p-1$, then $\varepsilon(4 / 3)=1 / 2$.

Finally, let $N$ be a large integer, and $n$ such that $2^{n} \leq N<2^{n+1}$. Then

$$
\begin{aligned}
|E \cap[1, N]| & \geq\left|E_{n_{0}+1}\right|+\cdots+\left|E_{n-1}\right| \geq \frac{c \tau}{4}\left[\left(n_{0}+1\right)+\cdots+(n-1)\right] \\
& \geq d_{0} n^{2} \geq d_{1}(\log N)^{2}
\end{aligned}
$$

where $d_{0}, d_{1}$ are positive constants. If now $E$ is a $p$-Rider set, we have the mesh condition $|E \cap[1, N]|=O\left((\log N)^{p /(2-p)}\right)$. This requires $2 \leq p /(2-p)$, that is $p \geq 4 / 3$. And this ends the proof of Theorem 2.7.

Remark. The third-named author proved the following (552], Lema 2.4) (which is actually implicitly already contained in [48], Lemme 7.2, Théorème 7.1, and Théorème 2.3 (iv)):

(*) For every finite subset $A \subseteq \mathbb{Z}$, there exists a quasi-independent subset $B \subseteq A$ such that $|B| \geq \delta\left(|A| / \psi_{A}\right)^{2}$, where $\delta>0$ is a numerical constant.

On the other hand, G. Pisier ([47], Lemme 5.2) proved:

$$
\mathbb{E}\left\|\sum_{k} a_{k} r_{k} e_{k}\right\|_{\Psi} \leq C\left(\sum_{k}\left|a_{k}\right|^{2}\right)^{1 / 2}
$$

where $C$ is a numerical constant, $\left(r_{k}\right)_{k}$ is the Rademacher sequence, and \|\|$_{\Psi}$ is the Orlicz space associated to $\Psi(x)=\mathrm{e}^{x^{2}}-1$.

Taking our selectors $\varepsilon_{k}$ with mean $\delta_{k}=c n / 2^{n}$ for $k \in I_{n}$, standard symmetrization and centering arguments give:

$$
\mathbb{E}\left\|\sum_{k \in I_{n}} \varepsilon_{k} e_{k}\right\|_{\Psi} \leq C \sqrt{n} .
$$

In other terms, we have, in view of Lemma 2.9

$$
\mathbb{E}\left(\psi_{\Lambda_{n}}\right) \leq C \sqrt{n} \leq C^{\prime}\left|\Lambda_{n}\right|^{1 / 2} .
$$

If we could prove a concentration inequality, variant of Lemma 1.3, then this variant and the Borel-Cantelli lemma would imply from (3) that:

$$
\text { Almost surely } \psi_{\Lambda_{n}} \leq C^{\prime \prime}\left|\Lambda_{n}\right|^{1 / 2} \text { for } n \text { large enough. }
$$

We could then combine $(*)$ and $(4)$ directly to obtain the following alternative proof of Theorem 2.7 Select $\omega \in \Omega$ such that $\Lambda$ is strongly ergodic, with $\left|\Lambda_{n}\right| \geq c n / 2$, and $\psi_{\Lambda_{n}} \leq C^{\prime \prime}\left|\Lambda_{n}\right|^{1 / 2} ;$ take then a quasi-independent set $E_{n} \subseteq \Lambda_{n}$ of size

$$
\left|E_{n}\right| \geq \delta\left(\frac{\left|\Lambda_{n}\right|}{\psi_{\Lambda_{n}}}\right)^{2} \geq \delta C^{\prime \prime-2}\left|\Lambda_{n}\right| \geq \delta^{\prime} n ;
$$

the set $E=\bigcup_{n} E_{n}$ then has the required properties. 
To end this section, we consider the case $p>4 / 3$. We cannot keep the property of uniform convergence $(C U C)$, nor that of being $q$-Sidon stated in Theorem 2.5. We do not know whether this is only due to the method. But being $p$-Rider with $p>4 / 3$ might be a rather weak condition (see [35] and [36]).

Theorem 2.10 For every $4 / 3 \leq p<2$ there exists a set $\Lambda$ of integers which is $p$-Rider, but is not $q$-Rider for $q<p$ and which is $\Lambda(q)$ for every $q<+\infty$, but which is uniformly distributed (so in particular dense in the Bohr group, and $\mathcal{C}_{\Lambda}$ contains $\left.c_{0}\right)$.

The proof is essentially the same as in Theorem 2.2, except that we take selectors $\varepsilon_{k}$ of mean

$$
\delta_{k}=c \frac{(\log k)^{\alpha}}{k(\log \log k)^{\alpha+1}} \quad \text { for } k \geq 1,
$$

where $\alpha=2(p-1) /(2-p) \geq 1$, and replace $M_{n}=n^{n}$ by the smallest integer $\geq n^{\beta n}$, with $\beta$ any number $>\alpha$ (for instance $\beta=\alpha+1$ ), which we call again $M_{n}$. The estimate:

$$
\mathbb{P}\left(\Omega_{n}\left(M_{n}\right)\right) \leq 2 \frac{c^{n}}{n^{n}} \frac{\left(\log M_{n}\right)^{n(\alpha+1)}}{M_{n}}
$$

still holds, and now gives:

$$
\mathbb{P}\left(\Omega_{n}\left(M_{n}\right)\right) \leq C^{\prime n} \frac{(\log n)^{n(\alpha+1)}}{n^{(\beta-\alpha) n}} .
$$

Then easy computations show that:

(*) Almost surely $\left|\Lambda_{M_{n}}\right| \sim(n \log n)^{\alpha+1}$ for $n$ sufficiently large;

(**) Almost surely $\left|\Lambda_{n}^{\prime}\right| \sim n^{\alpha}(\log n)^{\alpha+1}$ for $n$ sufficiently large.

Property $(*)$ guaranties that $\Lambda(\omega)$ will still be almost surely $p$-Rider, and $(* *)$ with the mesh condition implies that $\Lambda$ is not $q$-Rider for $q<p$.

The $\Lambda(q)$ property cannot be obtained by the Littlewood-Paley method, but follows from [43, Theorem 4.7.

\section{Large thin sets in prescribed sets of integers}

In this section, we start from a prescribed set $\Lambda_{0}=\left\{\lambda_{1}<\lambda_{2}<\ldots<\lambda_{N}<\right.$ $\ldots\}$ of positive integers, and randomly construct a thin set $\Lambda$ inside $\Lambda_{0}$ in the following way. We still have our selectors $\varepsilon_{1}, \ldots, \varepsilon_{N}, \ldots$ of respective means $\delta_{1}, \ldots, \delta_{N}, \ldots$ This time, however, we set

$$
\Lambda=\Lambda(\omega)=\left\{\lambda_{j} \in \Lambda_{0} ; \varepsilon_{j}(\omega)=1\right\},
$$

i.e. we select randomly some of the $\lambda_{j}$ 's, and ignore the other integers. Such constructions have been made previously by S. Neuwirth ([43]). 
We always assume that $\Lambda_{0}$ is ergodic, namely that

$$
A_{\Lambda_{0}, N}(t)=N^{-1}\left(\mathrm{e}^{i \lambda_{1} t}+\cdots+\mathrm{e}^{i \lambda_{N} t}\right) \underset{N \rightarrow+\infty}{\longrightarrow} l(t), \quad \forall t \in \mathbb{T} .
$$

In this context, we have the following theorem, which extends Bourgain's Theorem 1.10.

Theorem 3.1 ([43], Th. 5.4) Let $\Lambda_{0}$ be an ergodic (resp. strongly ergodic, resp. uniformly distributed) set of positive integers, and let $\varepsilon_{1}, \ldots, \varepsilon_{N}, \ldots$ be selectors with respective expectation $\delta_{1}, \ldots, \delta_{N}, \ldots$ with $\left(\delta_{n}\right)_{n \geq 1}$ decreasing. Assume that $\sigma_{N} / \log \lambda_{N} \underset{N \rightarrow+\infty}{\longrightarrow}+\infty$, where $\sigma_{N}=\delta_{1}+\cdots+\delta_{N}$. Then, almost surely, the set $\Lambda$ is ergodic (resp. strongly ergodic, resp. uniformly distributed). More precisely, if $A_{\Lambda_{0}, N}(t) \underset{N \rightarrow+\infty}{\longrightarrow} l(t)$, we have, almost surely, with $\Lambda_{N}=$ $\Lambda \cap\left\{\lambda_{1}, \ldots, \lambda_{N}\right\}$,

$$
A_{N}(t)=\frac{1}{\left|\Lambda_{N}\right|} \sum_{n \in \Lambda_{N}} e_{n}(t) \underset{N \rightarrow+\infty}{\longrightarrow} l(t), \quad \forall t \in \mathbb{T} .
$$

We sketch the proof. First, we require

Lemma 3.2 Let $\varepsilon_{1}, \ldots, \varepsilon_{N}$ be selectors of respective expectations $\delta_{1}, \ldots, \delta_{N}$. Setting $\sigma_{N}=\delta_{1}+\cdots+\delta_{N}$, one has the following inequality:

$$
\mathbb{P}\left(\left\|\sum_{k=1}^{N}\left(\varepsilon_{k}-\delta_{k}\right) e_{\lambda_{k}}\right\|_{\infty}>15 \sqrt{\sigma_{N} \log \lambda_{N}}\right) \leq 8 / N^{2},
$$

provided that $\sigma_{N} \geq 25 \log \lambda_{N}$.

Proof. Set $Q=\sum_{k=1}^{N}\left(\varepsilon_{k}-\delta_{k}\right) e_{\lambda_{k}}$. For fixed $t \in \mathbb{R}$, one has $Q(t)=\sum_{k=1}^{N} X_{k}$, where $X_{k}=e_{\lambda_{k}}(t)\left(\varepsilon_{k}-\delta_{k}\right)$. The $X_{k}$ 's are independent, bounded by 1 , and centered complex random variables; so, letting $t_{N}=5 \sqrt{\sigma_{N} \log \lambda_{N}}$, and using Lemma1.3. we get

$$
\begin{aligned}
\mathbb{P}\left(\|Q\|_{\infty}>3 t_{N}\right) & \leq \mathbb{P}\left(\sup _{t \in F_{N}}|Q(t)|>t_{N}\right) \leq \sum_{t \in F_{N}} \mathbb{P}\left(|Q(t)|>t_{N}\right) \\
& \leq 4\left|F_{N}\right| \exp \left(-\frac{t_{N}^{2}}{8 \sigma_{N}}\right)=8 \lambda_{N}^{1-25 / 8} \leq N^{1-25 / 8} \leq 8 N^{-2},
\end{aligned}
$$

where $F_{N}=\left\{j \pi / \lambda_{N} ; 0 \leq j \leq 2 \lambda_{N}-1\right\}$ is the set of the $\left(2 \lambda_{N}\right)^{t h}$ roots of unity, and where the first inequality follows from Bernstein inequality (see [22]).

Proof of Theorem 3.1. Notice first that

$$
\frac{1}{\sigma_{N}} \sum_{n=1}^{N} \delta_{n} e_{\lambda_{n}}(t) \underset{N \rightarrow+\infty}{\longrightarrow} l(t), \quad \forall t \in \mathbb{T} .
$$


In fact, set $E_{n}=e_{\lambda_{1}}(t)+\cdots+e_{\lambda_{n}}(t)$ and $l=l(t)$. Since $\left(\delta_{n}\right)_{n}$ is nonincreasing, two Abel's partial summations give:

$$
\begin{aligned}
\sum_{n=1}^{N} \delta_{n} e_{\lambda_{n}}(t) & =\sum_{n=1}^{N-1}\left(\delta_{n}-\delta_{n+1}\right) E_{n}+\delta_{N} E_{N} \\
& =\sum_{n=1}^{N-1} n l\left(\delta_{n}-\delta_{n+1}\right)+N l \delta_{N}+o\left(\sum_{n=1}^{N-1} n\left(\delta_{n}-\delta_{n+1}\right)+N \delta_{N}\right) \\
& =l \sigma_{N}+o\left(\sigma_{N}\right) .
\end{aligned}
$$

Setting $Q_{N}=\sum_{n=1}^{N}\left(\varepsilon_{n}-\delta_{n}\right) e_{\lambda_{n}}$, we have:

$$
\left\|A_{N}-\frac{1}{\sigma_{N}} \sum_{n=1}^{N} \delta_{n} e_{\lambda_{n}}\right\|_{\infty} \leq \frac{2\left\|Q_{N}\right\|_{\infty}}{\sigma_{N}},
$$

since

$$
\begin{aligned}
\| \frac{1}{S_{N}} \sum_{n=1}^{N} \varepsilon_{n} e_{\lambda_{n}} & -\frac{1}{\sigma_{N}} \sum_{n=1}^{N} \delta_{n} e_{\lambda_{n}} \|_{\infty} \\
& \leq\left|\frac{1}{S_{N}}-\frac{1}{\sigma_{N}}\right|\left\|\sum_{n=1}^{N} \varepsilon_{n} e_{\lambda_{n}}\right\|_{\infty}+\frac{1}{\sigma_{N}}\left\|\sum_{n=1}^{N}\left(\varepsilon_{n}-\delta_{n}\right) e_{\lambda_{n}}\right\|_{\infty} \\
& \leq \frac{\left|S_{N}-\sigma_{N}\right|}{\sigma_{N}}+\frac{\left\|Q_{N}\right\|_{\infty}}{\sigma_{N}}=\frac{\left|Q_{N}(0)\right|+\left\|Q_{N}\right\|_{\infty}}{\sigma_{N}} \leq \frac{2\left\|Q_{N}\right\|_{\infty}}{\sigma_{N}} .
\end{aligned}
$$

Now, Lemma 3.2 gives:

$$
\mathbb{P}\left(\left\|Q_{N}\right\|_{\infty}>15 \sqrt{\sigma_{N} \log \lambda_{N}}\right) \leq 8 \lambda_{N}^{-2} \leq 8 N^{-2}
$$

if $\sigma_{N} \geq 25 \log \lambda_{N}$; so we get, by the Borel-Cantelli lemma,

$$
\frac{\left\|Q_{N}\right\|_{\infty}}{\sigma_{N}}=O\left(\sqrt{\frac{\log \lambda_{N}}{\sigma_{N}}}\right)
$$

almost surely. In view of the hypothesis, we have:

$$
\left\|A_{N}-\frac{1}{\sigma_{N}} \sum_{n=1}^{N} \delta_{n} e_{\lambda_{n}}\right\|_{\infty} \underset{N \rightarrow+\infty}{\longrightarrow} 0 \quad \text { almost surely; }
$$

and so, almost surely $A_{N}(t) \underset{N \rightarrow+\infty}{\longrightarrow} l(t)$ for each $t$, which is the desired conclusion.

\subsection{Regularity}

Let $I$ be a finite interval of $\mathbb{N}^{*}$ and $\nu(I)=\left|\Lambda_{0} \cap I\right|$ be the number of indices $n$ for which $\lambda_{n} \in I$. In the sequel, we assume that $\Lambda_{0}$ has the following regularity property: 
There exists a continuous eventually strictly increasing function $\varphi:] 0,+\infty[\rightarrow] 0,+\infty[$ such that:

$$
\frac{\nu([N, 2 N[)}{\varphi(N)} \underset{N \rightarrow+\infty}{\longrightarrow} 1
$$

and:

$$
\frac{\varphi(2 x)}{\varphi(x)} \underset{x \rightarrow+\infty}{\longrightarrow} l>1 .
$$

Note that $l \leq 2$, since $\nu\left(\left[2^{k}, 2^{k+1}\left[\leq 2^{k}\right.\right.\right.$ implies that $(1-\varepsilon)^{k-k_{0}} l^{k-k_{0}} \varphi\left(2^{k-k_{0}}\right) \leq$ $\varphi\left(2^{k}\right) \leq(1+\varepsilon) 2^{k}$.

We say that $\Lambda_{0}$ is regular if these properties hold.

They are obviously verified when $\lambda_{n}=n^{s}$, and also, by the Prime Number Theorem, when $\lambda_{n}=p_{n}$, with $\varphi(x)=x / \log x$.

It is easy to see that (3.1) and (3.2) imply that $\Lambda_{0}$ has a polynomial growth, namely that there exist two constants, $a, d>0$ such that:

$$
\nu([1, k]) \geq a k^{d}
$$

(or, equivalently, $\lambda_{N} \leq a^{\prime} N^{1 / d}$ ).

It follows that the condition $\sigma_{N} / \log \lambda_{N} \underset{N \rightarrow+\infty}{\longrightarrow}+\infty$ of Theorem 3.1 reduces then to the previous condition $\sigma_{N} / \log N \underset{N \rightarrow+\infty}{\longrightarrow}+\infty$ of Theorem 1.10,

Moreover $\Lambda_{0}$ satisfies:

$$
\lambda_{8 n} \geq 2 \lambda_{n} \quad \text { for } n \geq 1 \text { large enough. }
$$

Indeed, if $\nu\left(\left[1,2^{k-1}[)<n \leq \nu\left(\left[1,2^{k}[)\right.\right.\right.\right.$, then $2 \lambda_{n} \leq 2^{k+1}$ and it suffices to show that $\nu\left(\left[2^{k-1}, 2^{k+1}[) \leq 7 n\right.\right.$. But

$$
\begin{aligned}
\nu\left(\left[2^{k-1}, 2^{k+1}[)\right.\right. & \leq(1+\varepsilon)\left(\varphi\left(2^{k-1}\right)+\varphi\left(2^{k}\right)\right) \leq(1+\varepsilon)^{2}\left(l^{2}+l\right) \varphi\left(2^{k-2}\right) \\
& \leq(1+\varepsilon)^{3}\left(l^{2}+l\right) \nu\left(\left[2^{k-2}, 2^{k-1}[) \leq(1+\varepsilon)^{3}\left(l^{2}+l\right) \nu\left(\left[1,2^{k-1}[)\right.\right.\right.\right. \\
& \leq 7 \nu\left(\left[1,2^{k-1}[) \leq 7 n\right.\right.
\end{aligned}
$$

for $\varepsilon>0$ small enough, and $n$ large enough.

As in Section 2, we restrict ourselves to selectors with mean $\delta_{n}=\alpha_{n} / n$, where $\left(\delta_{n}\right)_{n}$ decreases to 0 , and $\left(\alpha_{n}\right)_{n}$ tends to infinity, and moreover, except in the last theorem, $\left(\alpha_{n}\right)_{n}$ increases.

The following lemma is quite similar to Lemma 2.1. We indicate some changes which are needed, and how the regularity occurs.

Lemma 3.3 Let $s \geq 2$ and $M$ be integers and let

$\Omega_{s}(M)=\left\{\omega \in \Omega ; \Lambda(\omega) \cap\left[\lambda_{M},+\infty[\right.\right.$ contains at least a relation of length $s\}$.

We have, for s large enough,

$$
\mathbb{P}\left(\Omega_{s}(M)\right) \leq \frac{(16 \mathrm{e})^{s}}{s^{s}} \sum_{j>M} \delta_{j}^{2} \sigma_{j}^{s-2} .
$$


Proof. As in the proof of Lemma 2.1, we write $\Omega_{s}(M)=\bigcup_{l \geq M+s-1} \Delta_{l}$, where $\Delta_{l}$ is defined by

$\Delta_{l}=\left\{\omega ; \Lambda(\omega) \cap\left[\lambda_{M},+\infty[\right.\right.$ contains at least a relation of length $s$ and with greatest term $\left.\lambda_{l}\right\}$.

It suffices to show that

$$
\mathbb{P}\left(\Delta_{l}\right) \leq \frac{8^{s} 2^{s-2}}{(s-2) !} \delta_{l}^{2} \sigma_{l}^{s-2} .
$$

The proof proceeds as in Lemma2.1, replacing $i_{1}, \ldots, i_{s-1}$ and $l$ by $\lambda_{i_{1}}, \ldots, \lambda_{i_{s-1}}$ and $\lambda_{l}$ respectively. The relation (**) gives $\lambda_{i_{s-1}} \geq \lambda_{l} / s$. The regularity appears now to say that $i_{s-1} \geq l / 8^{s}$. Indeed, otherwise, by (3.4), we should have, for $s$ large enough,

$$
\lambda_{l}>\lambda_{8^{s} i_{s-1}} \geq 2^{s} \lambda_{i_{s-1}} \geq 2^{s} \frac{\lambda_{l}}{s}>\lambda_{l} .
$$

This gives the lemma since $\left(\alpha_{n}\right)_{n}$ increases:

$$
\delta_{i_{s-1}}=\frac{\alpha_{i_{s-1}}}{i_{s-1}} \leq \frac{\alpha_{l}}{i_{s-1}}=\frac{\alpha_{l}}{l} \frac{l}{i_{s-1}} \leq 8^{s} \delta_{l} .
$$

Since this basic lemma still holds for random subsets of prescribed sets $\Lambda_{0}$, the first main theorems of Section 2 still hold and their proofs requires only minor modifications because of Theorem 3.1. We therefore content ourselves with stating them.

Theorem 3.4 Let $\Lambda_{0}$ be a regular, strongly ergodic set of positive integers. There exists a set $\Lambda \subseteq \Lambda_{0}$ which is:

(1) $p$-Sidon for all $p>1, \Lambda(q)$ for all $q<+\infty$, CUC, but which is:

(2) strongly ergodic (in particular, $\mathcal{C}_{\Lambda}$ contains $c_{0}$ and $\Lambda$ is not a Rosenthal set).

Theorem 3.5 Let $\Lambda_{0}$ be as in the previous theorem, and let $1<p<4 / 3$. Then, there exists a set $\Lambda \subseteq \Lambda_{0}$ which is:

(1) strongly ergodic (in particular, $\mathcal{C}_{\Lambda}$ contains $c_{0}$ and so $\Lambda$ is not a Rosenthal set), but which is:

(2) a CUC-set, $\Lambda(q)$ for all $q<+\infty$, and

(a) is $p$-Rider, but is not $q$-Rider for $q<p$,

(b) is $q$-Sidon for all $q>p /(2-p)$.

Theorem 3.6 Same as in the previous theorem, but instead of property (a):

(a') $\Lambda$ is $q$-Rider for every $q>p$, but is not $p$-Rider. 
Theorem 3.7 Let $\Lambda_{0}=\left\{\lambda_{1}, \ldots\right\}$ be a regular, strongly ergodic set. Then, there exists a set $\Lambda \subseteq \Lambda_{0}$ which is strongly ergodic and contains a set $E$ which

(1) has a positive upper density in $\Lambda(\omega)$ (so in particular, $\mathcal{C}_{E}$ contains $c_{0}$ and $E$ is not a Rosenthal set), and

(2) is a CUC-set, is 4/3-Rider, but not $q$-Rider for $q<4 / 3$, and is a $\Lambda(q)$-set for all $q<+\infty$; more precisely, for all $q>2$, we have $\|f\|_{q} \leq C q^{2}\|f\|_{2}$ for all $f \in \mathcal{P}_{E}$, where $C>0$ is a numerical constant.

The proof is the same as that of Theorem 2.7, so we omit it. We merily note the following facts.

The sequence $\left(\delta_{k}\right)_{k}$ is eventually decreasing. Indeed, for $n \geq n_{\varepsilon}$, we have, by the regularity conditions (3.1) and (3.2), if $\varepsilon>0$ is chosen so that $(1-\varepsilon)^{2} l \geq 1$,

$$
\nu_{n+1} \geq(1-\varepsilon) \varphi\left(2^{n+1}\right) \geq(1-\varepsilon)^{2} l \varphi\left(2^{n}\right) \geq(1-\varepsilon)^{3} l \nu_{n} \geq \nu_{n} .
$$

Next, (3.1) implies that, for some constant $\alpha>0$, and for $2^{q}<N \leq 2^{q+1}$,

$$
\begin{aligned}
\sigma_{N}=\delta_{1}+\cdots+\delta_{N} & \geq \sum_{n=1}^{q}\left(\sum_{k \in I_{n}} \delta_{k}\right)=c \sum_{n=1}^{q} \log \nu_{n} \\
& \geq c \alpha \sum_{n=1}^{q} n \geq c(\alpha / 2) q^{2} .
\end{aligned}
$$

Since $\Lambda_{0}$ has polynomial growth: $\lambda_{N}=O\left(N^{1 / d}\right)$, we have $\log \lambda_{N} \leq \lambda_{2^{q+1}}=$ $O(q)$. It follows that:

$$
\sigma_{N} / \log \lambda_{N} \underset{N \rightarrow+\infty}{\longrightarrow}+\infty .
$$

Finally, we have to replace the parameter $\psi_{A}$ in the proof of Theorem 2.7 by:

$$
\psi_{A}^{\prime}=\sup _{p \geq 2} \frac{\left\|e_{A}^{\prime}\right\|_{p}}{\sqrt{p}}, \quad \text { where } e_{A}^{\prime}=\sum_{\lambda_{k} \in A} e_{\lambda_{k}} .
$$

Since we have, for any interval $I: \psi_{I}^{\prime} \leq C \nu(I) / \sqrt{\log \nu(I)}$, the rest of the proof will then work with no essential change.

Remark. Consider the $\psi$-parameter associated to the squares, that is:

$$
\psi_{N}^{\prime}=\sup _{q \geq 2} \frac{\left\|S_{N}\right\|_{q}}{\sqrt{q}},
$$

where $S_{N}(x)=\sum_{n=1}^{N} \mathrm{e}^{i n^{2} x}$. It follows from results of Zalcwasser ([61]), that we have very precise estimates on $\left\|S_{N}\right\|_{q}$ : there exist numerical constants $C_{1}, C_{2}>$ 0 such that:

$$
C_{1} N^{1-2 / q} \leq\left\|S_{N}\right\|_{q} \leq C_{2} N^{1-2 / q}
$$

whenever $q \geq 5$ and $N \geq 1$ (when $q$ is near 4 , a logarithmic factor $(\log N)^{1 / q}$ should be added in the upper estimate). Therefore, the a priori crude estimate used in the proof of Theorem 2.7 is, at least for the squares, optimal, as it is for the set of all the positive integers. 


\section{References}

[1] G.I. Arkipov and K.I. Oskolkov, On a special trigonometric series and its applications, Math. Sbornik 134 (1987), 145-155.

[2] R. Blei, Sidon partitions and p-Sidon sets, Pacific J. Math. 65 (1976), 307313.

[3] R. Blei, Fractional cartesian products of sets, Ann. Inst. Fourier 29 (1979), 79-105.

[4] R. Blei, Combinatorial dimension and certain norms in Harmonic Analysis, Amer. J. Math. 106 (1984), 847-887.

[5] R. Blei and T.W. Körner, Combinatorial dimension and random sets, Israel J. Math. 47 (1984), 65-74.

[6] J.R. Blum, B. Eisenberg and L.-S. Hahn, Ergodic theory and the measure of sets in the Bohr group, Acta Scient. Math. Szeged 34 (1973), 17-24.

[7] J. Bourgain, Propriétés de décomposition pour les ensembles de Sidon, Bull. Soc. Math. France 111 (1983), 421-428.

[8] J. Bourgain, Sidon sets and Riesz products, Ann. Inst. Fourier 35 (1985), 137-148.

[9] J. Bourgain, Subspaces of $L_{N}^{\infty}$, arithmetical diameter and Sidon sets, Probability in Banach Spaces V, Proceed. Medford 1984, Lecture Notes in Math. 1153 (1985), 96-127.

[10] J. Bourgain, On the maximal ergodic theorem for certain subsets of the integers, Israel J. Math. 61 (1988), 39-72.

[11] J. Bourgain, On the behavior of the constant in the Littlewood-Paley inequality, Geometric aspects of functional analysis, Isr. Semin., GAFA, Isr. 1987-88, Lecture Notes in Math. 1376 (1989), 202-208.

[12] J. Bourgain, Bounded orthogonal sets and the $\Lambda(p)$-set problem, Acta Math. 162 (1989), 227-246.

[13] J. Bourgain and V. Milman, Dichotomie du cotype pour les espaces invariants, C.R.A.S. Paris 300 (1985), 263-266.

[14] M. Bożejko and T. Pytlik, Some types of lacunary Fourier series, Colloq. Math. 25 (1972), 117-124.

[15] M. Déchamps-Gondim, Sur les compacts associés aux ensembles lacunaires, les ensembles de Sidon et quelques problèmes ouverts, Publications Mathématiques d'Orsay 84-01 (1984), exposé 7.

[16] J. Diestel, Sequences and Series in Banach Spaces, Graduate Texts in Math. 92, Springer-Verlag (1984). 
[17] S. Drury, Sur les ensembles de Sidon, C.R.A.S. Paris 271 (1970), 162-163.

[18] R.E. Edwards and K.A. Ross, p-Sidon sets, J. Funct. Anal. 15 (1974), 404427.

[19] A. Figà-Talamanca, An example in the theory of lacunary Fourier series, Boll. Unione Matem. Ital. 3 (1970), 375-378.

[20] J. Fournier, Two UC-sets whose union is not a UC-set, Proc. Amer. Math. Soc. 84 (1982), 69-72.

[21] J. Fournier and L. Pigno, Analytic and arithmetic properties of thin sets, Pacific J. Math. 105 (1983), 115-141.

[22] C. Frappier, Q.I. Rahman and St. Ruscheweyh, New inequalities for polynomials, Trans. Amer. Math. Soc. 288 (1985), 69-99.

[23] G.W. Johnson, Theorems on lacunary sets, especially $p$-Sidon sets, Studia Math. 58 (1976), 209-221.

[24] G.W. Johnson and G.S. Woodward, On $p$-Sidon sets, Indiana Univ. Math. J. 24 (1974), 161-167.

[25] J.-P. Kahane, Some random series of functions, Second ed., Cambridge Univ. Press, Cambridge (1985).

[26] B. Kashin and L. Tzafriri, On random sets of uniform convergence, Math. Notes 54 (1993), 677-687.

[27] Y. Katznelson, Suites aléatoires d'entiers, Lecture Notes in Math. 336, Springer-Verlag Berlin (1973), 148-152.

[28] Y. Katznelson and P. Malliavin, Vérification statistique de la conjecture de la dichotomie sur une classe d'algèbres de restriction, C.R.A.S. Paris 262 (1966), 490-492.

[29] S. Konyagin, On divergence of trigonometric Fourier series everywhere, C.R.A.S. Paris 329 (1999), 693-697.

[30] T.W. Körner, Fourier analysis, Cambridge University Press (1988).

[31] S. Kwapien and A. Pełczyński, Absolutely summing operators and translation invariant spaces of functions on compact abelian groups, Math. Nachrichten 94 (1980), 303-340.

[32] M. Ledoux and M. Talagrand, Probability in Banach spaces, Ergeb. Math. 23 Springer-Verlag (1991).

[33] P. Lefèvre, Sur les ensembles de convergence uniforme, Publ. Math. d'Orsay 94-24 (1994), 1-70.

[34] P. Lefèvre, Measures and lacunarity sets, Studia Math. 133 (1999), 145-161. 
[35] P. Lefèvre, D. Li, H. Queffélec, and L. Rodríguez-Piazza, Lacunary sets and function spaces with finite cotype, (submitted)

[36] P. Lefèvre and L. Rodríguez-Piazza, $p$-Rider sets are $q$-Sidon sets, (submitted)

[37] D. Li, A remark about $\Lambda(p)$-sets and Rosenthal sets, Proc. Amer. Math. Soc. 126 (1998) 3329-3333.

[38] J. Lindenstrauss and L. Tzafriri, Classical Banach Spaces I and II, Classics in Math., Springer (1997).

[39] J.M. Lopez and K.A. Ross, Sidon Sets, Marcel Dekker 13 (1975).

[40] F. Lust, Produits tensoriels injectifs d'espaces de Sidon, Colloq. Math. 32 (1975), 285-289.

[41] F. Lust-Piquard, Propriétés géométriques des sous-espaces invariants par translation de $L^{1}(G)$ et $\mathcal{C}(G)$, Sémin. Géom. Espaces Banach, Ecole Polytechnique, Paris (1977-78), Exposé n ${ }^{\circ} 26$.

[42] F. Lust-Piquard, Bohr local properties of $\mathcal{C}_{\Lambda}(\mathbb{T})$, Colloq. Math. 58 (1989), 29-38.

[43] S. Neuwirth, Random constructions inside lacunary sets, Annales Inst. Fourier 49 (1999), 1853-1867.

[44] K.I. Oskolkov, On spectra of uniform convergence, Soviet Math. Dokl. 33 (1986), 616-620.

[45] L. Pedemonte, Sets of uniform convergence, Colloq. Math. 33 (1975), 123132.

[46] G Pisier, Ensembles de Sidon et espaces de cotype 2, Séminaire sur la géométrie des espaces de Banach 1977-1978, Ecole Polytechnique, Paris (1978), exposé 14 .

[47] G. Pisier, Sur l'espace de Banach des séries de Fourier aléatoires presque sûrement continues, Séminaire sur la géométrie des espaces de Banach 1977-1978, Ecole Polytechnique, Paris (1978), exposés 17-18.

[48] G. Pisier, De nouvelles caractérisations des ensembles de Sidon, Math. Anal. and Applic., Part B, Advances in Math. Suppl. Studies, Vol 7B (1981), 685-726.

[49] D. Rider, Gap series on groups and spheres, Canad. J. Math. 18 (1966), 389-398.

[50] D. Rider, Randomly continuous functions and Sidon sets, Duke Math. J. 42 (1975), 759-764. 
[51] L. Rodríguez-Piazza, Caractérisation des ensembles p-Sidon p.s., C.R.A.S. Paris 305 (1987), 237-240.

[52] L. Rodríguez-Piazza, Rango y propiedades de medidas vectoriales. Conjuntos $p$-Sidon p.s., Thesis, Universidad de Sevilla (1991).

[53] H.P. Rosenthal, On Trigonometric Series Associated with Weak* Closed Subspaces of Continuous Functions, Journ. Math. Mech. 17 (1967), 485490.

[54] W. Rudin, Trigonometric Series with Gaps, Journal of Math. and Mech. 9 (1960), 203-227.

[55] I. Singer, Bases in Banach Spaces I, Springer Verlag (1970).

[56] P.M. Soardi and G. Travaglini, On sets of completely uniform convergence, Colloq. Math. 45 (1981), 317-320.

[57] G. Travaglini, Some properties of UC-sets, Boll. Unione Matem. Ital. 15 (1978), 272-284.

[58] N.T. Varopoulos, Une remarque sur les ensembles de Helson, Duke Math. J. 43 (1976), 387-390.

[59] P. Wojtaszczyk, Banach Spaces for Analysts, Cambridge University Press (1991).

[60] G. S. Woodward, $p$-Sidon Sets and a Uniform Property, Indiana Univ. Math. Journal 25 (1976), 9951-1003.

[61] Z. Zalcwasser, Polynômes associés aux fonctions modulaires $\vartheta$, Studia Math. 7 (1938), 16-35.

[62] A. Zygmund, Trigonometric Series, Second Ed., Vol. I \& II, Cambridge Math. Library, Cambridge Univ. Press (1993). 